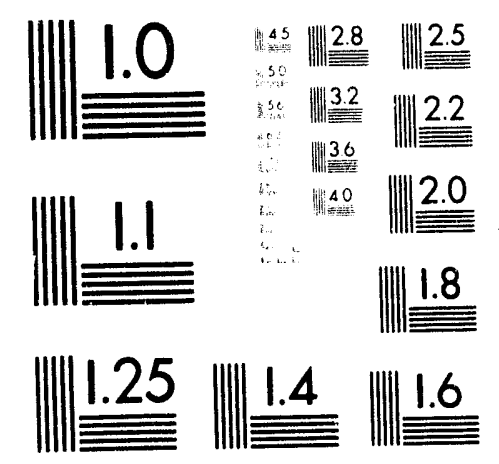



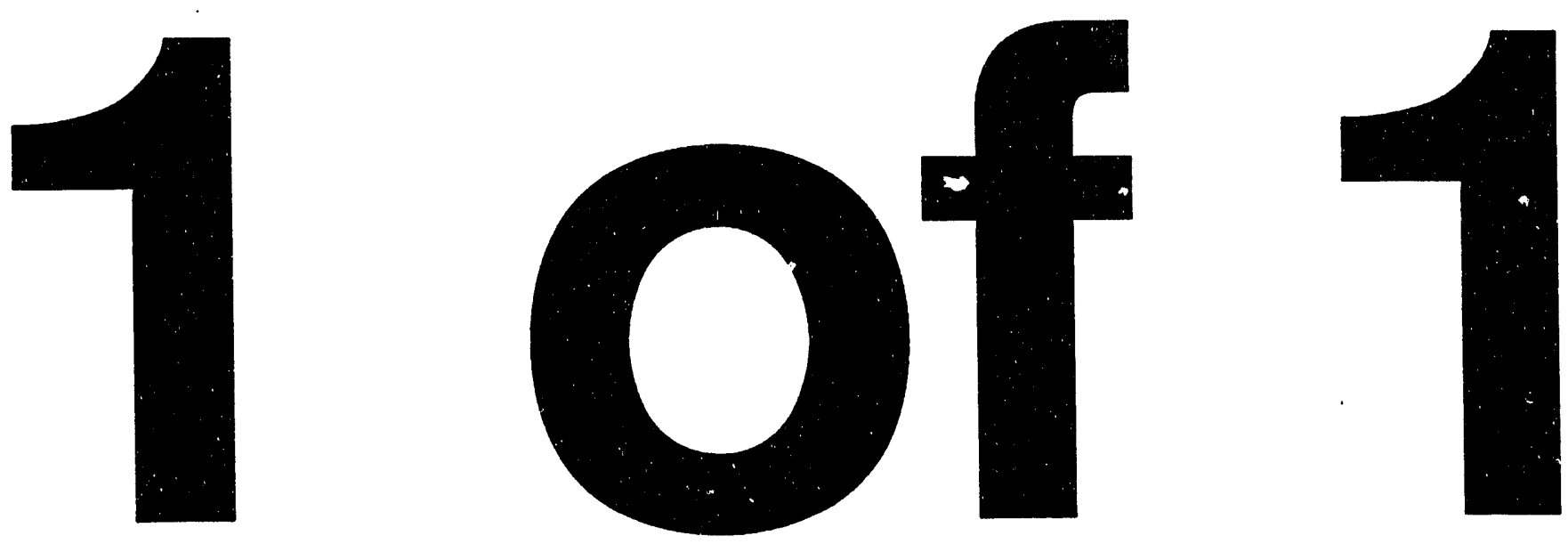


\title{
Momentum and Coordinate Space Three-nucleon Potentials
}

\author{
S. A. Coon \\ Physics Department \\ New Mexico State University \\ Las Cruces, NM 88003, USA
}

\author{
M. T. Peña \\ CEBAF, Theory Group \\ 12000 Jefferson Ave, \\ Newport News, VA 23606, USA*.
}

June 10,1993

\begin{abstract}
In this paper we give explicit formulae in momentum and coordinate space for the threenucleon potentials due to $\rho$ and $\pi$ meson exchange, derived from off-mass-shell meson-nucleon scattering amplitudes which are constrained by the symmetries of QCD and by the experimental data. Those potentials have already been applied to nuclear matter calculations. Here we display additional terms which appear to be the most important for nuclear structuie. The potentials are decomposed in a way that separates the contributions of different physical mechanisms involved in the meson-nucleon amplitudes. The same type of decomposition is presented for the $\pi-\pi$ TM force: the $\Delta$ isobar, the chiral symmetry breaking and the nucleon pair terms are isolated.
\end{abstract}

\section{DISCLAIMER}

This report was prepared as an account of work sponsored by an agency of the United States Government. Neither the United States Government nor any agency thereof, nor any of their employees, makes any warranty, express or implied, or assumes any legal liability or responsibility for the accuracy, completeness, or usefulness of any information, apparatus, product, or process disclosed, or represents that its use would not infringe privately owned rights. Reference herein to any specific commercial product, process, or service by trade name, trademark, manufacturer, or otherwise does not necessarily constitute or imply its endorsement, recommendation, or favoring by the United States Government or any agency thereof. The views and opinions of authors expressed herein do not necessarily state or reflect those of the United States Government or any agency thereof.

\footnotetext{
•Permanent address: Centro de Física Nuclear, 1699 Lisboa Codex, Portugal.
}

\section{¿ECEIVEL AU6 251993 OSTI}




\section{Introduction}

Three-nucleon potentials based on $\pi$ and $\rho$ meson exchange have been derived from different underlying approaches. The recent papers which include $\rho$ exchange $[1,2,3,4]$ tend to extend to the rho meson whichever approach had been already used for the two pion exchange potential considered earlier (because of its longer range). The early history of the $\pi-\pi$ three-nucleon potential was categorized in this way and summarized in [5]. Those potentials built upon the excitation of the nucleon into a $\Delta$ isobar were the first and contain the least physical input. These $\Delta$ mediated potentials have recently been extended to include $\rho$ exchange in Refs. [1] and [4]. Soon after the first $\Delta$ mediated $\pi-\pi$ potential was constructed, it was realized that chiral symmetry (breaking) must be incl." od in the potential. That is, the theoretical $\pi \mathrm{N}$ amplitudes must satisfy well-defined chiral (so cá "soft pion") limits [6]. One way to accomplish this begins with the use of (effective) Lagrangians with pions, nucleons, deltas etc. which satisfy approximate chiral symmetry [7, 8, 9]. This approach has recently been extended to include $\rho$ mesons, so that the assumed Lagrangians must also obey gauge invariance [2]. This approach has built in the correct symmetries but the couplings must be estimated and, furthermore, the results have not really been tested against the pion-nucleon data $[10]$.

Alternatively, the approach used in the Tucson-Melbourne (TM) family of forces is based upon applying the Ward identities of current algebra to axial-vector nucleon scattering. The Ward identities are saturated with nucleon and $\Delta(1230)$ poles. Then employing PCAC (partial conservation of the axial-vector current), one can derive expressions for the on-mass-shell pion-nucleon scattering amplitudes which map out satisfactorily the empirical coefficients of the Höhler subthreshold crossing symmetric expansion based on dispersion relations [11]. The off-mass-shell extrapolation (needed for the exchange of virtual, spacelike pions in a nuclear force diagram) of the most important amplitude $\bar{F}^{+}$can be written in a form which depends on measured on-shell amplitudes only. This rewriting of the amplitude to bury all reference to models of the $\Delta$ exploits a convenient correspondence between the structure of the terms corresponding to spontaneously broken chiral symmetry and the structure of the model $\Delta$ term. However, both the field theoretic [12] and dispersion theoretic [13] $\Delta$ contributions to $\bar{F}^{+}$result in an equally excellent description of the data [14], so the early emphasis on maximum model independence can perhaps be relaxed. This will be done in the present paper.

Extending this current algebra-PCAC program to the Compton-like processes [15] $\gamma_{\nu}+N \rightarrow$ $A_{\mu}+N$, where $\gamma_{\nu}$ is a vector photon and $A_{\mu}$ is an axial-vector current, provides, through vector dominance, a solid basis for modeling a $\rho-\pi$ exchange three-nucleon force. Again the amplitude 
is obtained via Ward identities but this time exploiting in addition the gauge condition on the off-shell electroproduction amplitude. Unfortunately, there is no empirical subthreshold expansion of the invariant amplitudes of pion photo- and electroproduction. Instead, the amplitudes are tested against the soft pion theorems and (on-shell) against multipoles at threshold or slightly above threshold. The amplitude which forms the basis of the TM $\rho-\pi$ force meets these tests [15]. including the challenge of the recently measured neutral pion photoproduction [16].

The so-called Tucson-Melbourne (TM) family of three-nucleon forces, in the particular case of $\rho-\pi$ and $\rho-\rho$ exchange, has never been displayed in a form suitable for applications in general nuclear systems (in particular, we have in mind few-nucleon systems). In the work of Ref. [3] those potentials were applied to nuclear matter where certain approximations could be carried out. As a result, Ref. [3] failed to provide a simultaneously complete and convenient explicit form of the T.M $\rho-\pi$ and $\rho-\rho$ forces, ready for immediate use in any calculation, as we propose to present in this paper.

Nevertheless, the nuclear matter calrulation of Ref. [3] was indicative of the role of the $\rho$ meson in screening the $\pi$ exchange effect. The same effect had already been seen in the nuclear matter calculation of Ref. [1] where a three-nucleon force constructed only from $N N-N \Delta$ transition potentials was used.

Furthermore, calculations of the triton bound state with inclusion of the TM $2 \pi$ exchange potential yield over-binding and strong dependence on the $\pi N N$ form factor regulator mass [17. 18]. This reflects the presence in the $\pi-\pi$ TM force of a term (usually denoted in the published literature as the "c" term [5]) in part due to chiral symmetry breaking and responsible for the rapid variation of the lower-order terms in the $\pi-N$ amplitude, as demanded by the soft pion theorems. It appears then natural to extend the calculations on the three-nucleon system to the inclusior of the TM $\rho-\pi$ and $\rho-\rho$ forces hoping to cancel part of the $\pi-\pi$ force effect, in a similar fashion to what happens in the two-nucleon interactions. Moreover, since the leading terms of the expansions of the the $\rho N \rightarrow \pi N$ and $\rho N \rightarrow \rho N$ amplitudes do not have to build in a drastic variation in the low energy region, they do not have such short range singularities. In this spirit, the Sendai group included a $\rho-\pi$ force in a triton calculation [19] which, however, due to lack of a consistent body of information in the literature, mixes several terms and parameter prescriptions of more than one origin.

Another motivation to have $\rho-\pi$ and $\rho-\rho$ forces derived from the same principles used in the construction of the TM $\pi-\pi$ force is to have available a more complete two-meson-exchange threenucleon interaction, which is needed to draw conclusions about the role played by the delta-isobar in the force. Only after introducing the consistent full family of forces in the hamiltonian, does 
it become legitimate to make comparisons with the Hannover (two-body $N N \rightarrow N \Delta$ transition potential) approach and judge how much effect comes from true $\Delta$-isobar propagation. A first study of this question, restricted however to the use of the TM $\pi-\pi$ force, appeared most recently in the literature [20]. Another one is in preparation where the $\rho-\pi$ and $\rho-\rho$ TM force input is needed $[21,22]$. In the context of these recent developments in few-body calculations, the present paper supplies the necessary information to address the one-decade unsolved problem of comparing and constrasting the Hannover with the Tucson-Melbourne approaches.

Finally, and aiming beyond the triton bound state problem, the stage of nowadays powerful calculations allows the information of this paper to be applied to 1) the present concentrated effort on experimental searches for direct evidence of three-nucleon forces in the three-nucleon continuum guided by the three nucleon-scattering ralculations of the Bochum group [23]; and 2) the $\alpha$ particle wavefunction calculation [24], under the strong indications from existing calculations $[25,26,27,28]$ that the three-nucleon force effects, when fully considered, will be relevant in bringing the binding energy closer to its experimental value.

\section{The $\pi-\pi$ Potentials}

In this section we carry out the program of splitting the $\pi-\pi$ force into the contributions relative to the different physical processes underlying the $\pi \mathrm{N}$ amplitude. Historically the current algebra amplitudes were given in a form which emphasized their model independent character. That was achieved by not disentangling explicitely the $\Delta$ contribution from the chiral symmetry breaking terms. Such an attitude was justified by allowing a more direct relation to empirical quantities, where that separation could not be seen. The process of undoing this way of displaying the force is implicit in the set of initial papers $[5,29]$. Here we merely summarize the procedure by showing the main steps.

The $\pi-\pi$ force was derived from a non-relativistic reduciion of the Feynman diagram of Fig. 1. We begin by defining the $T$-matrix for the three-nucleon process depicted in Fig. 1 in terms of the $S$-matrix:

$$
S_{f i}-\delta_{f i}=-(2 \pi)^{4} i \delta^{(4)}\left(\sum p_{f}-\sum p_{i}\right) N_{f} N_{i} T_{f i}
$$

where $N_{f}=\prod_{j}\left(1 / 2 E_{j}\right)^{1 / 2}(2 m)^{n_{f} / 2}$ (the index $\mathrm{j}$ labelling all the particles in the final state and $n_{f}$ being the number of fermions) and $N_{i}$ (defined similarly) are the normalization factors necessary to make $T_{f i}$ the covariant $T$-matrix. Then the matrix elements of the momentum space three-nucleon 


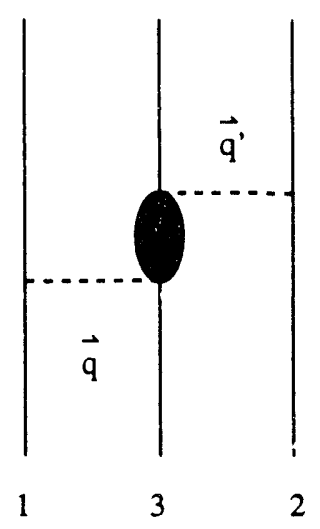

Figure 1: Diagram for the $\pi-\pi$ force

potential $W$ are given by the non-relativistic reduction of the three body $S-1$ :

$<\vec{p}_{2} \cdot \vec{n}_{2}{ }_{2}{\overrightarrow{p^{\prime}}}_{3}|S-1| \vec{p}_{1} \vec{p}_{2} \vec{p}_{3}>_{N R}=-i(2 \pi) \delta\left(p_{10}^{\prime}-p_{20}^{\prime}-p_{30}^{\prime}-p_{10}-p_{20}-p_{30}\right)<{\overrightarrow{p^{\prime}}}_{1}{\overrightarrow{p^{\prime}}}_{2}{\overrightarrow{p^{\prime}}}_{3}|W| \vec{p}_{1} \vec{p}_{2} \vec{p}_{3}>$,

so that

$$
\left.<\overrightarrow{p_{1}^{\prime}}, \overrightarrow{p_{2}^{\prime}}, \overrightarrow{p_{3}^{\prime}}|W| \overrightarrow{p_{1}}, \overrightarrow{p_{2}}, \overrightarrow{p_{3}}\right\rangle=(2 \pi)^{3} \delta^{3}\left(\overrightarrow{p_{1}^{\prime}}+\overrightarrow{p_{2}^{\prime}}+\overrightarrow{p_{3}^{\prime}}-\overrightarrow{p_{1}}-\overrightarrow{p_{2}}-\overrightarrow{p_{3}}\right)<\overrightarrow{p_{1}^{\prime}} \overrightarrow{p_{2}^{\prime}} \vec{p}_{3}^{\prime}\left|T_{N R}\right| \vec{p}_{1} \vec{p}_{2} \vec{p}_{3}>
$$

We note that this choice for the normalization corresponds to the use of Dirac spinors normalized as $\bar{u} u=1$ and to momentum eigenstates such that $\left\langle\vec{p} \mid \overrightarrow{p^{\prime}}\right\rangle=(2 \pi)^{3} \delta\left(\vec{p}-\overrightarrow{p^{\prime}}\right)$. Most momentum space three-body codes normalize the momentum space eigenstates to the Dirac delta function without the factor of $(2 \pi)^{3}$; this point is discussed rather extensively in Ref. [29]. Our choice of convention does not affect the coordinate space formulae as it is (correctly) absorbed into the Fourier transform, such that $\left\langle\vec{r} \mid \overrightarrow{r^{\prime}}\right\rangle=\delta\left(\vec{r}-\overrightarrow{r^{\prime}}\right)$. The full three-nucleon potential $W$ is the sum of three cyclic terms $W_{1}, W_{2}$, and $W_{3}$ where $W_{3}$ has particle 3 in the middle (active nucleon) as in Figure 1. The other two terms are obtained by cyclic permutation of the indices of the incoming nucleons.

Now we turn our attention to the intermediate state depicted as a blob in Fig.1. It is described by the (two-body) $\pi \mathrm{N}$ scattering amplitude $\pi^{j}(q)+N(p) \rightarrow \pi^{i}\left(q^{\prime}\right) N\left(p^{\prime}\right)$ written in terms of invariant amplitudes $F$ and $B$ which have the the most general isospin decomposition:

$$
T^{i j}=-\bar{u}\left(p^{\prime}, s^{\prime}\right)\left\{\delta^{i j}\left(F^{+}-\frac{B^{+}}{4 m}\left[\boldsymbol{d}, \phi^{\prime}\right]\right)+i \epsilon^{i j k}\left(F^{-}-\frac{B^{-}}{4 m}\left[\boldsymbol{d}, \boldsymbol{d}^{\prime}\right]\right)\right\} u(p, s)
$$


To proceed, one removes the nucleon pole from the four off-shell crossing symmetric invariants $F^{ \pm}\left(\nu, t, q^{2}, q^{\prime 2}\right)$ and $B^{ \pm}\left(\nu, t, q^{2}, q^{2}\right)$. Given the four background $\pi \mathrm{N}$ amplitudes (obtained after the nucleon pole is removed) $\vec{F}^{ \pm}\left(\nu, t, q^{2}, q^{\prime 2}\right)$ and $\bar{B}^{ \pm}\left(\nu, t, q^{2}, q^{\prime 2}\right)$, where $\nu=\left(q+q^{\prime}\right) \cdot\left(p+p^{\prime}\right) /(4 m)$, $t=\left(q-q^{\prime}\right)^{2}$, one performs an expansion in the pion four momenta $q, q^{\prime}$. In this expansion, after terms of the order of $(\mu / m)^{2}$ ( $\mu$ and $m$ stand for the pion and the nucleon mass respectively) or higher are dropped, only the (t-channel) isospin even non-spin flip $\bar{F}^{+}$and the isospin odd spin-flip $\bar{B}^{-}$amplitudes survive $[5,29]$. We will concentrate then on those two. The non-spin flip even current algebra amplitude is:

$$
\bar{F}^{+}\left(\nu, t, q^{2}, q^{\prime 2}\right)=f\left(\nu, t, q^{2}, q^{\prime 2}\right) \frac{\sigma}{f_{\pi}^{2}}+C^{+}\left(\nu, t, q^{2}, q^{\prime 2}\right)
$$

where $\sigma$ is the pion-nucleon $\sigma$ term and $f_{\pi} \approx 93 \mathrm{MeV}$. The double divergence $q^{\prime} \cdot \bar{M}^{+} \cdot q / f_{\pi}^{2}$ of the background axial vector amplitude denoted by $C^{+}$contains the higher order $\Delta$ isobar contribution. This amplitude is given by $[13,5]$

$$
\begin{aligned}
C^{+}\left(\nu, t, q^{2}, q^{\prime 2}\right) & =\frac{2 g^{* 2}}{9 m^{2}} \frac{\nu_{\Delta} \alpha\left(q^{\prime} \cdot q\right)}{\nu_{\Delta}^{2}-\nu^{2}} \\
& -\frac{g^{* 2}(M+m)}{9 M^{2} m}\left[(M-m)(M+m)^{2}+\left(q^{2}+q^{\prime 2}\right)(2 M+m)\right. \\
& \left.-q^{\prime} \cdot q\left(2 M-m+\left(q^{2}+q^{\prime 2}\right)(M+m)^{-1}\right)\right],
\end{aligned}
$$

where $g^{*} \approx 1.82 \mu^{-1}$ is the $\pi \Delta \mathrm{N}$ coupling, $M=8.825 \mu$ the $\Delta$ mass, $m=6.726 \mu$ the nucleon mass, $\nu_{\Delta}=\left(M-m^{2}-q^{\prime} \cdot q\right) / 2 m, \alpha\left(q^{\prime} \cdot q\right)=\left(E_{\pi}^{2}-q^{\prime} \cdot q\right)\left[(M+m)^{2}-q^{\prime} \cdot q\right]+\left(\bar{\mu}^{2}-q^{\prime} \cdot q\right)\left[(M+m) E_{\pi}-\frac{1}{2}\left(\ddot{\mu}^{2}+q^{\prime} \cdot q\right)\right]$ with $E_{\pi}=\left(M-m^{2}+\bar{\mu}^{2}\right) / 2 M$ the center of mass energy of the pion at the $\Delta$ resonance. Here we have taken $\bar{\mu}^{2}=1 / 2\left(q^{2}+q^{\prime 2}\right)$ which reduces to $\mu^{2}$ when both pions are on mass shell.

In general, $C^{+}$must have the simple form $[13,5]$

$$
C^{+}\left(\nu, t, q^{2}, q^{2}\right)=c_{1} \nu^{2}+c_{2} q \cdot q^{\prime}+O\left(q^{4}\right)
$$

On the other hand, the assumed form of the function $f$,

$$
f\left(\nu, t, q^{2}, q^{\prime 2}\right)=(1-\beta)\left(\frac{q^{2}+q^{2}}{\mu^{2}}-1\right)+\beta\left(\frac{t}{\mu^{2}}-1\right)
$$

(adapted [5, 14] for $\pi \mathrm{N}$ scattering from the $S U(3)$ generalization of the Weinberg low energy expansion for $\pi \pi$ scattering [30]) is such that $\bar{F}^{+}$does satisfy the soft pion theorems

$$
\bar{F}^{+}(0,0,0,0)=-\frac{\sigma}{f_{\pi}^{2}}
$$




$$
\bar{F}^{+}\left(0, \mu^{2}, 0, \mu^{2}\right)=\bar{F}^{+}\left(0, \mu^{2}, \mu^{2}, 0\right)=0,
$$

where $C^{+}$vanishes identically, and (with the aid of Eq. (7)) the constraint at the (on-shell and measurable) Cheng-Dashen point:

$$
\bar{F}^{+}\left(0,2 \mu^{2}, \mu^{2}, \mu^{2}\right)=\frac{\sigma}{f_{\pi}^{2}}+\mathcal{O}\left(q^{4}\right) .
$$

In contrast to meson-meson scattering, $\beta$ in ( 8 ) is not determined by soft-meson theorems (because the nucleon four momenta cannot be taken soft) and is to be extracted from experiment. The most recent data analysis from the Karlsruhe group [11] makes this extraction slightly dependent on $\mathrm{t}$ : $\beta$ varies from 0.46 (for $t=0$ ) to 0.52 (for $t=\mu^{2}$ ). This situation is different from the situation of older data $[5,13,31]$ which was consistent with an almost constant $\beta$ value of 0.4 in the same range of $t$.

Neglecting the $\nu^{2}$ and $q_{0}$ terms in (5) because they are of the order of $(\mu / m)^{2}$ or higher, the $\vec{F}^{+}$amplitude can be expanded in the three-vector pion momenta $\vec{q}$ and $\overrightarrow{q^{\prime}}$ as follows:

$$
\bar{F}^{+}\left(0, t, q^{2}, q^{2}\right)=-\frac{\sigma}{f_{\pi}^{2}}+\left(\frac{\sigma}{f_{\pi}^{2}} \frac{2 \beta}{\mu^{2}}-c_{2}\right) \vec{q} \cdot \overrightarrow{q^{\prime}}-\frac{\sigma}{\mu^{2} f_{\pi}{ }^{2}}\left(\vec{q}^{2}+{\overrightarrow{q^{\prime}}}^{2}\right)
$$

The last equation explicitly exhibits the separation between the (higher order in $\vec{q}^{2}$ ) $\Delta$ contribution - contained in the $c_{2}$ term alone - and the remaining chiral symmetry breaking terms. In ref. [5] and subsequent discussions of the TM $\pi-\pi$ force, the $c_{2}$ and $\beta$ constants in the coefficient of the $\vec{q} \cdot \overrightarrow{q^{\prime}}$ term were eliminated in favor of the on-shell (measurable) quantity $\vec{F}^{+}\left(0, \mu^{2}, \mu^{2}, \mu^{2}\right)$

$$
\bar{F}^{+}\left(0, \mu^{2}, \mu^{2}, \mu^{2}\right)=(1-\beta) \frac{\sigma}{f_{\pi}^{2}}+\frac{c_{2} \mu^{2}}{2}-
$$

According to Eq. (12), the $\Delta$ term alone does not bring a structure in momentum space different from the chiral symmetry ureaking term in $\vec{q} \cdot \overrightarrow{q^{\prime}}$. To isolate the $\Delta$ contribution one simply evaluates the constant $c_{2}$, which, from (7) (and taking both pions on-mass-shell), can be done by evaluating the derivative of (6) with respect to $q \cdot q^{\prime}$, at $t=2 \mu^{2}\left(q \cdot q^{\prime}=0\right)$ and $\nu=0$. The result is $\hat{\imath}_{2}=-1.54 \mu^{-3}$. This coefficient coincides with twice $C^{+}$evaluated at $t=\mu^{2}\left(q \cdot q^{\prime}=\mu^{2} / 2\right)$ and $\nu=0$ and compares well with the value $-1.58 \mu^{-1}$, obtained for the total contribution from $C^{+}$at $t=0\left(q \cdot q^{\prime}=\mu^{2}\right)$ and $\nu=0$. The two last comparisons give an idea of the negligible importance of the $O\left(q^{4}\right)$ terms in Eq. (7).

From the $\pi \mathrm{N}$ amplitude in conjunction with the $\pi \mathrm{NN}$ vertices and pion propagators, one constructs the three body $T_{N R}$, which according to Eq. (3) defines the three-body force represented in Fig. $1[5,29]$. 
The constant $c_{2}$ contributes then to the overall coefficient "b" that has been used in nuclear calculations $\left(b=b_{\sigma}+b_{\Delta} ; b_{\Delta}=c_{2}\right)$

$$
b=-\frac{\sigma}{f_{\pi}^{2}} \frac{2 \beta}{\mu^{2}}+c_{2}=-\frac{2}{\mu^{2}}\left(\frac{\sigma}{f_{\pi}^{2}}-\bar{F}^{+}\left(0, \mu^{2}, \mu^{2}, \mu^{2}\right)\right)
$$

The value $b=-2.58 \mu^{-3}$, obtained from early experimental results [31] $\bar{F}^{+}\left(0, \mu^{2}, \mu^{2}, \mu^{2}\right)=-0.16 \mu^{-1}$ and $\sigma / f_{\pi}^{2}=1.13 \mu^{-1}$ implies a chiral symmetry breaking coefficient $b_{\sigma}=b-c_{2}=-1.04 \mu^{-3}$. If the updated set of Karlsruhe data is taken instead, $\bar{F}^{+}\left(0, \mu^{2}, \mu^{2}, \mu^{2}\right)=-0.28 \mu^{-1}$ and $\sigma / f_{\pi}^{2}=1.03 \mu^{-1}$, then $b=-2.62 \mu^{-3}$ and consequently $b_{\sigma}=-1.08 \mu^{-3}$. The comparison between the numbers $b_{\Delta}$ and $b_{\sigma}$ exhibits that the $\Delta$ terms do not dominate the amplitude and any description which does not obey chiral symmetry breaking [32] is insufficient.

The isolation of the $\Delta$ from the broken chiral symmetry terms being complete, we have to turn to the backward-propagating nucleon Born term that is added to the background amplitude, to generate the three-body force. That term is calculated by subtracting the forward time-ordered propagating nucleon term from the covariant nucleon poles. Such a subtraction was done in refs. $[5,29]$. Dropping again terms that are of the order of $(\mu / m)^{2}$, the backward-propagating nucleon term $F_{Z}^{+}$("Z-graph") is

$$
F_{Z}^{+}=\frac{g^{2}}{4 m^{3}}\left(\vec{q}^{2}+{\overrightarrow{q^{\prime}}}^{2}\right)
$$

where $\mathrm{g}$ is the pseudo-scalar $\pi \mathrm{NN}$ coupling constant. Since $F_{Z}^{+}$shows the same momentum dependence as the third term in Eq. (12), the constant $c_{z}=-g^{2} / 4 m^{3}=-0.15 \mu^{-3}$ contributes, together with the coefficient of the last term of Eq. (12), to the overall constant " $c$ " $\left(c=c_{\sigma}+c_{z}\right)$ that multiplies the $\vec{q}^{2}+{\overrightarrow{q^{\prime}}}^{2}$ term, in the definition of the TM $\pi-\pi$ three-body force:

$$
c=\frac{\sigma}{\mu^{2} f_{\pi}^{2}}-\frac{g^{2}}{4 m^{3}}+F_{\pi N N}^{\prime}(0) \frac{\sigma}{f_{\pi}^{2}}
$$

We note that the term proportional to $F_{\pi N N}^{\prime}(0)$ did not appear before in Eq. (12). This term nevertheless is inserted in $c$ because both the backward propagating part of the nucleon pole $F_{Z}^{+}$ and the $\Delta$ couple with the pion with a (assumed the same) form factor $F_{\pi N N}\left(q^{2}\right)$ which is defined as $g\left(q^{2}\right)=g F_{\pi N N}\left(q^{2}\right)$. The chiral breaking $\sigma$ term is a c-number [14] and has no intrinsic $q^{2}$ dependence (although it is multiplied by $f\left(\nu, t, q^{2}, q^{2}\right)$ ). It is convenient, if not necessary, however, since part of the amplitude is due to $F^{+}$and $C^{+}$, to multiply the final amplitude by form factors, dependent upon $q^{2}$ and $q^{2}$. Consequently, the constant term $\left(\sigma / f_{\pi}{ }^{2}\right.$, labeled "a" in the literature) attains a spurious momentum dependence from the form factors. The term proportional to $F_{\pi N N}^{\prime}(0)$ in Eq. (16) is inserted to correct for this spurious momentum dependence to the orders in $q^{2}$ and $q^{2}$ kept in the amplitude. 
The value $c=c_{\sigma}+c_{z}=1.0 \mu^{-3}$ that has been used before changes slightly to $c=0.91 \mu^{-3}$, with the new determination of $\sigma / f_{\pi}^{2}$. From these numbers one concludes that in the non-spinflip amplitude amplitude the chiral symmetry breaking term dominates the backward-propagating nucleon term.

The spin-flip current algebra amplitude $\bar{B}^{-}$is simpler to decompose into the physical contributions. This amplitude is $[13,5]$

$$
\bar{B}^{-}\left(\nu, t, q^{2}, q^{\prime 2}\right)=\left(F_{1 V}(t)+F_{2 V}(t)\right) \frac{1}{2 f_{\pi}^{2}}-\frac{g^{2}}{2 m^{2}}+D^{-}\left(\nu, t, q^{2}, q^{2}\right),
$$

where the function $D^{-}$contains the higher order $\Delta$ contribution and is given in references $[5,13,29]$. After the non-relativistic reduction of the $\pi \mathrm{N} \mathrm{T}$ matrix is done, in the process of doing the $\mu / \mathrm{m}$ expansion to derive the three-body-force, we note that $\bar{B}^{-}$is multiplied by $\left[\phi, \phi^{\prime}\right]$ which is already of second order in $\mu / m$, so we need only keep the first term in the expansion of (17). Therefore, the electromagnetic isovector form factors are approximated by a constant value

$$
F_{1 V}+F_{2 V}=1 .+3.70+\mathcal{O}\left(\frac{\mu^{2}}{m^{2}}\right)
$$

and only the constant term $\left(D_{0}=4.87 \mu^{-2}\right)$ in the expansion of $D^{-}$is kept. Taking into account the multiplicative factor $-1 / 2 m$ from Eq. (4), the $\Delta$ contributes with a coefficient $d_{\Delta}=D_{0} / 2 m=$ $-0.36 \mu^{-3}$. The remaining contribution, due to the two first terms of Eq. (17), is $d_{c a}=-0.24 \mu^{3}$. The sum $d_{4}=d_{c a}+d_{\Delta}=-0.60 \mu^{-3}$ defines the $d_{4}$ parameter that one encounters in the published equations for the force.

Finally the $B \bar{Z}$ contribution from the backward propagating nucleon pole ("Z-graph") is given to zeroth order [5] by

$$
B_{\bar{Z}}^{-}=\frac{g^{2}}{2 m^{2}}+\mathcal{O}\left(\frac{\mu^{2}}{m^{2}}\right)
$$

Traditionally then these disparate contributions have been added into a total " $\mathrm{d}$ " coefficient, $d=$ $d_{4}+d_{z}$, where $d_{4}$ has already been defined and $d_{z}=-B_{\bar{Z}} / 2 m$ singles out the pair term contribution. The constant $d_{z}$ has the same value as the pair term coefficient $d_{z}=c_{z}=-0.15 \mu^{-3}$ of the $F^{+}$ amplitude. The total " $\mathrm{d}$ " term coefficient becomes $d=d_{c a}+d_{\Delta}+d_{z}=-0.75 \mu^{-3}$. Again the numbers indicate that the pairing term is small relative to the background amplitude and although the $\Delta$ is important, it is only half of the " $d$ " coefficient.

Now that we have undone the usual representation of the Tucson-Melbourne $\pi-\pi$ three-body force, let us close by recalling the form of the amplitude $\pi N \rightarrow \pi N$,

$$
T=T_{Z}+\Delta T+q^{\prime} \cdot \bar{M} \cdot q
$$


which best expresses the degree of model dependence of the amplitudes. Here $T_{Z}$ stands for the "Z-graph" contribution, $T_{Z}=T_{B}-T_{F P B}$, where $T_{B}$ is the covariant Born term and $T_{F P B}$ is the forward propagating (positive energy) nucleon term. The model independent $\Delta T$ is added to the full Born term $T_{B}$ so that to leading order $T_{B}+\Delta T$ satisfies the low energy theorems (Ward identity constraints) exemplified by (9) and (10), and $q^{\prime} \cdot \bar{M} \cdot q$ is a background term for which (isobar) models are necessary. The grouping of the isobar contributions into $\Delta T+q^{\prime} \cdot \bar{M} \cdot q$ in Eq.( 20) enforces the largest degree of model independence of the off-shell amplitude. This is because to lowest order (all) isobar contributions are included in $\Delta T$, and $q^{\prime} \cdot \bar{M} \cdot q$ is constructed to give contributions only in higher order terms. These general statements apply equally to the $\rho-\pi$ and the $\rho-\rho$ amplitudes discussed later on.

What is unique about the $\pi \mathrm{N} \bar{F}^{+}$amplitude is that the background term $q^{\prime} \cdot \bar{M} \cdot q$ of Eq. ( $(\overrightarrow{)})$ has just the right structure to combine nicely with the momentum dependence (8) of $\Delta T$ so that the entire off-shell amplitude can be obtained directly from data without a specific reference to the $\Delta$ contributions to either $\Delta T$ or to $q^{\prime} \cdot \bar{M} \cdot q$. This point, while giving us greater confidence in the three-body force derivation, has lead to considerable frustration among those interested in three-nucleon forces. Hence the deconstruction above which is summarized in the following table.

\begin{tabular}{ccccc} 
& $T_{Z}$ & $\Delta T$ & $q^{\prime} \cdot \bar{M}_{\Delta} \cdot q$ & Total \\
\hline$\mu \mathrm{a}$ & 0 & +1.03 & 0 & +1.03 \\
$\mu^{3} \mathrm{~b}$ & 0 & -1.08 & -1.54 & -2.62 \\
$\mu^{3} \mathrm{c}$ & -0.15 & +1.06 & 0 & +0.91 \\
$\mu^{3} \mathrm{~d}$ & -0.15 & -0.24 & -0.36 & -0.75
\end{tabular}

Table 1: Expansion coefficients of the $\pi \mathrm{N}$ amplitude used in the $\pi-\pi$ force. Units of charged pion masses. $T_{Z}$ is the "Z-graph" contribution, $\Delta T$ is the model independent part needed to satisfy the low energy theorems, $q^{\prime} \cdot \bar{M} \cdot q$ is the background term for which (isobar) models are necessary.

The table organizes vertically the coefficients according to the type of momentum and spinisospin structure which ultimately they multiply. Within each row, the decomposition acording to $(20)$, is provided. The model independent terms labeled $\Delta T$ in the table arise from current algebra but are of two different types. In the first three rows (labeled $a, b, c$ ) they contain the pion-nucleon sigma term, an axial current-axial charge commutator which is a measure of chiral symmetry breaking. The last row (labeled $\mathrm{d}$ and originated by $\bar{B}^{-}$) contains a $\Delta T$ which is also a current-current commutator. However in this case the algebra is closed by the electromagnetic form factor of the nucleon and has nothing to do with chiral symmetry breaking. As we shall see 
in subsequent sections, the chiral symmetry breaking contributions to the $\Delta T$ of the $\rho N \rightarrow \pi . Y$ amplitute are quite small and, of course, absent altogether in the $\rho N \rightarrow \rho N$ amplitude obtained via vector dominance from $\gamma N \rightarrow \gamma N$.

The specific momentum and spin-isospin structure of the final three-nucleon force is provided in momentum space in Ref. [29] and coordinate space in Ref. [33], and will not be repeated here. The strength constants of the $2 \pi$ exchange three-body force are given in units of the charged pion mass $(139.6 \mathrm{MeV})$. The potentials, however, use an isospin formalism instead of charge states so it would seem natural to employ the $\mathrm{SU}(2)$ average pion mass $\left(2 m_{\pi^{+}}+m_{\pi^{0}}\right) / 3=138 \mathrm{MeV}$ in the propagators and form factors. 


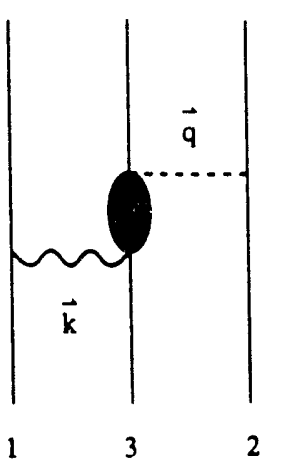

(a)

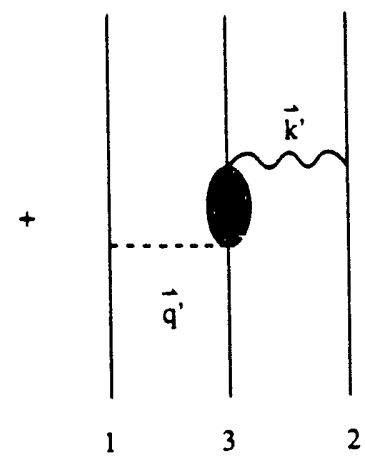

(b)

Figure 2: Diagrams for the $\pi-\rho$ force

\section{The $\rho-\pi$ Potentials}

The working definition of the $\rho-\pi$ three-nucleon potential is, as in the $\pi-\pi$ case, Eq. (3) written in section 2 . The three-body transition matrix $T_{N R}$ pictured in figure 2 contains now two different meson propagators together with the $\pi \mathrm{NN}$ and the $\rho \mathrm{NN}$ vertices from the external nucleonic lines of the diagram, in conjunction with the $\rho N \rightarrow \pi N$ intermediate amplitude.

The amplitude is modeled through vector dominance from the amplitude $\gamma N \rightarrow \pi N$ which is obtained by the method of Ward identities [15]. Those identities are (single and double) divergence conditions that determine the terms of the amplitude that are constant or linear in the exchanged momentum. Of the several spin-isospin components of the $\rho-\pi$ three nucleon potential only a few appear to be numerically significant. They correspond to the leading terms of the low energy expansion of $\rho N \rightarrow \pi N$ truncated to terms of second order, as was the $\pi-\pi$ force, in powers of the exchanged three-momenta between the nucleons. The ( $t$-channel) isotopic spin decomposition of the amplitude is the same as that of the $\pi \mathrm{N}$ amplitude of Eq. (4) because the $\rho$ is an isotriplet as well as the pion. Again, the expansion can be rearranged into the form of Eq. (20) for an optimal display of its degree of model independence. The isospin even model-independent terms $T_{Z}^{+}+\Delta T^{+}$nearly cancel to leading order because $\Delta T^{+}$goes to the soft pion $(q \rightarrow 0) \rho$ analog Fubini- Furlan-Roe stti limit of pion photoproduction $[15,16]$. The total model independent term in the $k \rightarrow 0, q \rightarrow 0$ limit takes on the form $1-m g_{A}(0) /\left(f_{\pi} g\right)$ which is of the order of few percent (Goldberger-Treiman discrepancy[34]). This cancellation is lessened away from the expansion point at zero, but then the model-dependent (and $\Delta$ dominated) background term $q^{\prime} \cdot \bar{M} \cdot q$ appears to 
totally dominate the $\rho N \rightarrow \pi N$ amplitude. Therefore, in the isospin even amplitude, only the $\Delta$-contribution, obtained in a dispersive sense, is developed into a $\rho-\pi$ potential in this paper. The situation is just the opposite for the model independent terms $T_{Z}^{-}+\Delta T^{-}$of the isospin odd amplitude. Here the low energy theorem of pion-photoproduction is due to Kroll and Ruderman and is simply the "Z-graph" $T_{\bar{Z}}^{-}$in pseudoscalar coupling [15]. This leading order (constant) term from the the $\rho$-analog Kroll- Ruderman theorem as $q \rightarrow 0, k \rightarrow 0$ should then form the basis of an important part of the $\rho-\pi$ potential. It must be supplemented, however, by the largest $\rho-\pi$ potential terms arising from the $\Delta$-dominated isospin odd background. Potential terms other than the above from $\Delta T^{ \pm}$, including the chiral symmetry breaking terms, appear to be much smaller than these just mentioned and will be neglected in the following (for details see Ref. [3]).

We now pick out the terms with one $\rho$ and one $\pi$ from Figure $2 \mathrm{a}$ which are believed to dominate the expansion and write them in detail. Those terms correspond to the "Z-graph" and the seemingly largest terms from the $\Delta$ isobar. The contribution from Figure $2 b$ follows from $2 a$ with the substitutions $1 \leftrightarrow 2$ and $\vec{k} \leftrightarrow-\vec{q}$ where $\vec{k}$ is the three-momentum of the rho and $\vec{q}$ the threemomentum of the pion. (In what follows the notation is slightly different from that of ref. [3] but closer to existing codes and practices).

\section{1 "Kroll-Ruderman" $\rho-\pi$ potential}

The analogue of the Kroll-Ruderman term of pion-photoproduction forms the basis of a specific local contribution to the overall $\rho-\pi$ exchange three-body potential that presents a first order dependence on the exchanged mesons momentum $\vec{k}$ and $\vec{q}$. It is consequently a contribution that is expected to compete strongly with the delta-isobar terms that are quadratic in the momentum.

This contribution comes from the t-channel isospin odd term in the $\rho N \rightarrow \pi N$ amplitude. When the pseudoscalar coupling for the $\pi N N$ vertex is assumed, this term is the backward-propagating nucleon Born term. In a form that evidences sequentially from left to right the role of the four vertices involved in the diagram, we can write for its momentum space representation

$$
\begin{aligned}
\left\langle\overrightarrow{r_{1}^{\prime}}, \overrightarrow{p_{2}^{\prime}}, \overrightarrow{p_{3}^{\prime}}\left|\mathcal{W}_{\rho \pi}^{-}(3 A)\right| \overrightarrow{p_{1}}, \overrightarrow{p_{2}}, \overrightarrow{p_{3}}\right\rangle= & +(2 \pi)^{3} \frac{\delta^{3}\left(\overrightarrow{p_{1}^{\prime}}+\overrightarrow{p_{2}^{\prime}}+\overrightarrow{p_{3}^{\prime}}-\overrightarrow{p_{1}}-\overrightarrow{p_{2}}-\overrightarrow{p_{3}}\right)}{\left(\overrightarrow{q^{2}}+\mu^{2}\right)\left(\overrightarrow{k^{2}}+m_{\rho}^{2}\right)} \\
& i \overrightarrow{\tau_{1}} \cdot \overrightarrow{\tau_{2}} \times \overrightarrow{\tau_{3}} i \overrightarrow{\sigma_{1}} \times \vec{k} \cdot \overrightarrow{\sigma_{3}} \overrightarrow{\sigma_{2}} \cdot \vec{q} \\
& \frac{g_{\rho}^{2}}{16 m^{3}}\left(F_{1 \rho}\left(k^{2}\right)+\kappa_{\rho} F_{2 \rho}\left(k^{2}\right)\right) F_{1 \rho}\left(k^{2}\right) g^{2} F_{\pi N N}^{2}\left(q^{2}\right)
\end{aligned}
$$

From this form it is evident that the coupling of the $\rho$ meson to the inner "blob" is proportional to $F_{1 \rho}\left(k^{2}\right)$, in accordance with the low energy theorem of Kroll and Ruderman. Also, to obtain 
Eq. (21) we kept the distinction between the Dirac and the Pauli couplings of the $\rho$ meson to the outer nucleon, which appears as the sum $F_{1 \rho}\left(k^{2}\right)+\kappa_{\rho} F_{2 \rho}\left(k^{2}\right)$. Subsequently, we retain in all equations one form-factor $F_{1 \rho}\left(k^{2}\right)$ for the Dirac coupling of the rho to the nucleon and another form-factor $F_{2 \rho}\left(k^{2}\right)$ for the Pauli coupling. The latter is multiplied by the on-mass-shell $\kappa_{\rho}$ which is taken to have the Hohler and Peitarinen value 6.6[35]. Separate form factors are not usual in nuclear physics, where it is common to take the same form for both the charge and the magnetic momentum form factors. Our choice has the advantage of being general and flexible to any type of (independent) behavior of the two form factors that experiment may eventually reveal. The price we have to pay is the introduction of a new parameter relative to $F_{2 \rho}\left(k^{2}\right)$.

The coupling of the pion to the nucleon is described by a form-factor $F_{\pi N N}$. Other form factors are introduced as needed. All form factors are normalized to unity at the meson on-mass-shell momentum. As for the structure, $\overrightarrow{\sigma_{2}} \cdot \vec{q}$ is the non-relativistic coupling of the $\pi$ to one of the outer nucleon lines, while $\overrightarrow{\sigma_{1}} \times \vec{k}$ comes from the $\rho$ coupling to the other external nucleon.

In coordinate space the "Kroll-Ruderman" potential becomes

$$
\begin{aligned}
\left\langle\overrightarrow{r_{1}^{\prime}}, \overrightarrow{r_{2}^{\prime}}, \overrightarrow{r_{3}^{\prime}}\left|\mathcal{W}_{\rho \pi}^{-}(3 A)\right| \overrightarrow{r_{1}}, \overrightarrow{r_{2}}, \overrightarrow{r_{3}}\right\rangle= & \delta^{3}\left(\overrightarrow{x_{1}^{\prime}}-\overrightarrow{x_{1}}\right) \delta^{3}\left(\overrightarrow{x_{2}^{\prime}}-\overrightarrow{x_{2}}\right) \delta^{3}\left(\overrightarrow{x_{3}^{\prime}}-\overrightarrow{x_{3}}\right) \\
& \frac{-\mu m_{\rho}}{(4 \pi)^{2} 16 m^{3}} g^{2} g_{\rho}^{2} \overrightarrow{\tau_{1}} \cdot \overrightarrow{\tau_{2}} \times \overrightarrow{\tau_{3}}\left(\overrightarrow{\sigma_{1}} \times \nabla_{1} \cdot \overrightarrow{\sigma_{3}}\right) \overrightarrow{\sigma_{2}} \cdot \nabla_{2} \\
& \left(\left(Z_{D D \rho}\left(x_{13}\right)+\kappa_{\rho} Z_{P D_{\rho}}\left(x_{13}\right)\right) Z_{\mu}\left(x_{23}\right)\right.
\end{aligned}
$$

where $\vec{x}_{i j} \equiv \overrightarrow{x_{i}}-\overrightarrow{x_{j}}$ and $\nabla_{i}^{j} \equiv \nabla_{i 3}^{j} \equiv \partial / \partial x_{i 3}^{j} \equiv \frac{x_{i 3}^{j}}{\left|x_{i 3}\right|} d / d x_{i 3},(j=1,2,3$ specifies a given cartesian component and $i=1,2$ a given nucleon).

The veralized form factor $H\left(\vec{q}^{2}\right)$ in each dimensionless coordinate space function $Z_{\mu}\left(x_{i j}\right)$ is typically oduct of monopole form factors: one for the vertex to the outer nucleon and a second is included i. ne coupling of the meson to the "blob" of figure 2. The coordinate space "propagator" has the generic form

$$
Z_{\alpha \beta \mu}(x)=\frac{4 \pi}{\mu} \int \frac{d^{3} q}{(2 \pi)^{3}} \frac{H_{\alpha \beta}\left(\vec{q}^{2}\right)}{\vec{q}^{2}+\mu^{2}} e^{\imath \vec{q} \cdot \vec{x}}
$$

where

$$
H_{\alpha \beta}\left(\vec{q}^{2}\right)=\frac{\Lambda_{\alpha}^{2}-\mu^{2}}{\Lambda_{\alpha}^{2}+\vec{q}^{2}} \frac{\Lambda_{\beta}^{2}-\mu^{2}}{\Lambda_{\beta}^{2}+\vec{q}^{2}} .
$$

For the exchanged pion $H_{\alpha \beta}\left(\vec{q}^{2}\right)=F_{\pi N N}^{2}\left(q^{2}\right)$ so that $\Lambda_{\alpha}=\Lambda_{\beta}$ and the $\alpha, \beta$ indices are suppressed. The $\rho$ propagator with subscript "DD $\rho$ " also indicates $\Lambda_{\alpha}=\Lambda_{\beta}$ with values from the Dirac coupling 
$\left(F_{1 \rho}\left(k^{2}\right)\right)^{2}$ and the transformation $\mu \rightarrow m_{\rho}$ but the subscript "PD $\rho$ " indicates $\Lambda_{\alpha} \neq \Lambda_{\beta}$ with values arising from the product of $F_{2 \rho}\left(k^{2}\right)$ and $F_{1 \rho}\left(k^{2}\right)$ and $\mu \rightarrow m_{\rho}$.

With these form factors and carrying out the indicated derivatives, the "Kroll-Ruderman" potential becomes

$$
\begin{aligned}
\left\langle\overrightarrow{r_{1}^{\prime}}, \overrightarrow{r_{2}^{\prime}}, \overrightarrow{r_{3}^{\prime}}\left|\mathcal{W}_{\rho \pi}^{-}(3 A)\right| \overrightarrow{r_{1}}, \overrightarrow{r_{2}}, \overrightarrow{r_{3}}\right\rangle= & \frac{-\mu m_{\rho}}{(4 \pi)^{2} 16 m^{3}} g^{2} g_{\rho}^{2} \delta^{3}\left(\overrightarrow{x_{1}^{\prime}}-\overrightarrow{x_{1}}\right) \delta^{3}\left(\overrightarrow{x_{2}^{\prime}}-\overrightarrow{x_{2}}\right) \delta^{3}\left(\overrightarrow{x_{3}^{\prime}}-\overrightarrow{x_{3}}\right) \\
& \overrightarrow{\tau_{1}} \cdot \overrightarrow{\tau_{2}} \times \overrightarrow{\tau_{3}}\left(\overrightarrow{\sigma_{1}} \times \hat{x}_{13} \cdot \overrightarrow{\sigma_{3}}\right) \overrightarrow{\sigma_{2}} \cdot \hat{x}_{23} \\
& \left(\left(X_{1 D D}^{\rho}\left(x_{13}\right)+\kappa_{\rho} X_{1 P D}^{\rho}\left(x_{13}\right)\right) X_{1}^{\mu}\left(x_{23}\right)\right.
\end{aligned}
$$

where $X_{1}(x)=Z^{\prime}(x)$. Explicitly for $\Lambda_{\alpha}=\Lambda_{\beta}=\Lambda$

$$
X_{1}^{\mu}(x)=-\mu\left[G(\mu x)-\frac{\Lambda^{2}}{\mu^{2}} G(\Lambda x)-\frac{1}{2}\left(\frac{\Lambda^{2}}{\mu^{2}}-1\right) e^{-(\Lambda x)}\right]
$$

and for $\Lambda_{\alpha} \neq \Lambda_{\beta}$

$$
X_{1 \alpha \beta}^{\mu}(x)=-\mu\left[G(\mu x)-\left(\frac{\Lambda_{\beta}}{\mu}\right)^{2}\left(\frac{\Lambda_{\alpha}^{2}-\mu^{2}}{\Lambda_{\alpha}^{2}-\Lambda_{\beta}^{2}}\right) G\left(\Lambda_{\beta} x\right)+\left(\frac{\Lambda_{\alpha}}{\mu}\right)^{2}\left(\frac{\Lambda_{\beta}^{2}-\mu^{2}}{\Lambda_{\alpha}^{2}-\Lambda_{\beta}^{2}}\right) G\left(\Lambda_{\alpha} x\right)\right]
$$

where

$$
G(x)=\frac{e^{-x}}{x}\left(1+\frac{1}{x}\right) .
$$

Note that the generic $X_{1}(x)=Z^{\prime}(x)$ has dimensions of mass and the spin operators are coupled with unit radial vectors. Then the overall constant has units of $\mathrm{MeV}$ and the magnitude is 28.72 $\mathrm{MeV}$. It should be clear that for rho exchange the generic $X_{1}^{\mu}(x)$ is written as $\mu \rightarrow m_{\rho}$. This finishes the explicit display of the "Kroll-Ruderman" term. It is expected to be the most important of the model-independent parts of the rho-pi potential.

\section{2 $\rho \pi$ Potential with $\Delta$ intermediate state}

\subsubsection{Isospin even amplitude}

Of the eight spin functions the potential decomposes into, the terms proportional to $1+\kappa_{\rho}$ are believed to be the largest because $\kappa_{\rho}$ has the value 6.6 on the rho-mass-shell. Those terms which are (t-channel) isospin even are generated from the spin functions $\overrightarrow{\sigma_{1}} \cdot \vec{q} \overrightarrow{\sigma_{2}} \cdot \vec{q}$ and $\overrightarrow{\sigma_{1}} \cdot \vec{k} \overrightarrow{\sigma_{2}} \cdot \vec{q}$. They take the momentum space form (see Eq. 2.19a of ref. [3].):

$$
\left.<\overrightarrow{p_{1}^{\prime}}, \overrightarrow{p_{2}^{\prime}}, \overrightarrow{p_{3}^{\prime}}\left|\mathcal{W}_{\rho \pi \Delta}^{+}(3 A)\right| \overrightarrow{p_{1}}, \overrightarrow{p_{2}}, \overrightarrow{p_{3}}\right\rangle=-(2 \pi)^{3} \frac{\delta^{3}\left(\overrightarrow{p_{1}^{\prime}}+\overrightarrow{p_{2}^{\prime}}+\overrightarrow{p_{3}^{\prime}}-\overrightarrow{p_{1}}-\overrightarrow{p_{2}}-\overrightarrow{p_{3}}\right)}{\left(\vec{q}^{2}+\mu^{2}\right)\left(\vec{k}^{2}+m_{\rho}^{2}\right)}
$$




$$
\begin{aligned}
& \overrightarrow{\tau_{1}} \cdot \overrightarrow{\tau_{2}}\left(\vec{k} \cdot \vec{k} \overrightarrow{\sigma_{1}} \cdot \vec{q} \overrightarrow{\sigma_{2}} \cdot \vec{q}-\vec{k} \cdot \vec{q} \overrightarrow{\sigma_{1}} \cdot \vec{k} \overrightarrow{\sigma_{2}} \cdot \vec{q}\right) \\
& \frac{1}{48 m^{5}} g_{\rho}\left(F_{1 \rho}\left(k^{2}\right)+\kappa_{\rho} F_{2 \rho}\left(k^{2}\right)\right) G_{M \rho}^{*} F_{\rho N \Delta}\left(k^{2}\right) \\
& \frac{m}{M} \frac{5 M-m}{M-m} g F_{\pi N N}\left(q^{2}\right)\left(m g^{*} F_{\pi N \Delta}\left(q^{2}\right)\right)
\end{aligned}
$$

where $M$ is the mass of the Delta and $m$ is the nucleon mass. The two terms can be combined as $\left(\overrightarrow{\sigma_{1}} \times \vec{k}\right) \cdot(\vec{q} \times \vec{k}) \overrightarrow{\sigma_{2}} \cdot \vec{q}$ which manifests the $\rho \mathrm{NN}$ coupling to "outer" nucleon 1 and the $\pi \mathrm{NN}$ coupling to "outer" nucleon 2. On the other hand, we note that the second term of Eq. 29 has the same structure $\left(\overrightarrow{\sigma_{1}} \cdot \vec{k} \overrightarrow{\sigma_{2}} \cdot \vec{q} \vec{k} \cdot \vec{q}\right)$ as the "b" term of the $2 \pi$ exchange potential.

In coordinate space the potential becomes

$$
\begin{aligned}
\left\langle\overrightarrow{r_{1}^{\prime}}, \overrightarrow{r_{i}^{\prime}} ;\left|\mathcal{W}_{\rho \pi \Delta}^{+}(3 A)\right| \overrightarrow{r_{1}}, \overrightarrow{r_{2}}, \overrightarrow{r_{3}}\right\rangle= & \delta^{3}\left(\overrightarrow{x_{1}^{\prime}}-\overrightarrow{x_{1}}\right) \delta^{3}\left(\overrightarrow{x_{2}^{\prime}}-\overrightarrow{x_{2}}\right) \delta^{3}\left(\overrightarrow{x_{3}^{\prime}}-\overrightarrow{x_{3}}\right) \\
& \frac{-\mu m_{\rho}}{(4 \pi)^{2} 48 M m^{4}} g g_{\rho} \frac{5 M-m}{M-m} G_{M \rho}^{*}\left(m g^{*}\right) \\
& \overrightarrow{\tau_{1}} \cdot \overrightarrow{\tau_{2}}\left[\overrightarrow{\nabla_{1}} \cdot \overrightarrow{\nabla_{1}} \overrightarrow{\sigma_{1}} \cdot \overrightarrow{\nabla_{2}} \overrightarrow{\sigma_{2}} \cdot \overrightarrow{\nabla_{2}}-\overrightarrow{\nabla_{1}} \cdot \overrightarrow{\nabla_{2}} \overrightarrow{\sigma_{1}} \cdot \overrightarrow{\nabla_{1}} \overrightarrow{\sigma_{2}} \cdot \overrightarrow{\nabla_{2}}\right] \\
& \left(\left(Z_{D G \rho}\left(x_{13}\right)+\kappa_{\rho} Z_{P G_{\rho}}\left(x_{13}\right)\right) Z_{N \Delta_{\mu}}\left(x_{23}\right)\right.
\end{aligned}
$$

where subscripts " $D G \rho$ " refer to the generalized form factor arising from the product of $F_{1 \rho}\left(k^{2}\right)$ and the $\rho \Delta N$ form factor $F_{\rho N \Delta}\left(k^{2}\right)$, " $P G \rho$ " to the analogous product $F_{2 \rho}\left(k^{2}\right) F_{\rho N \Delta}\left(k^{2}\right)$, and "N $\nu \mu$ " to $F_{\pi N N}\left(q^{2}\right) F_{\pi N \Delta}\left(q^{2}\right)$.

The second derivatives of Eq. (30) are most conveniently carried out with the aid of the following basic identity

$$
\gamma_{i} \partial_{j} Z(x)=\frac{1}{3}\left[\delta_{i j} Y_{2}(x)+\left(3 \hat{x}_{i} \hat{x}_{j}-\delta_{i j}\right) X_{2}(x)\right]
$$

$\left(\hat{x_{i}}=\overrightarrow{x_{i}}\left|x_{i}\right|\right)$ where $X_{2}(x)=Z^{\prime \prime}(x)-1 / x Z^{\prime}(x)$, and $Y_{2}(x)=Z^{\prime \prime}(x)+2 / x Z^{\prime}(x)$. Explicitly they are

$$
\begin{aligned}
& X_{2}^{\mu}(x)=\mu^{2}\left[F(\mu x)-\frac{\Lambda^{3}}{\mu^{3}} F(\Lambda x)-\frac{1}{2} \frac{\Lambda}{\mu} \Lambda x\left(\frac{\Lambda^{2}}{\mu^{2}}-1\right) G(\Lambda x)\right] \\
& Y_{2}^{\mu}(x)=\mu^{2}\left[\frac{e^{-\mu x}}{\mu x}-\frac{\Lambda^{3}}{\mu^{3}} \frac{e^{-\Lambda x}}{\Lambda x}-\frac{1}{2} \frac{\Lambda}{\mu} \Lambda x\left(\frac{\Lambda^{2}}{\mu^{2}}-1\right)\left(1-\frac{2}{\Lambda x}\right) \frac{e^{-\Lambda x}}{\Lambda x}\right] .
\end{aligned}
$$

Because

$$
F(x)=\frac{e^{-x}}{x}\left(1+\frac{3}{x}+\frac{3}{x^{2}}\right)
$$

for the seldom needed $\Lambda_{\alpha}=\Lambda_{\beta}=\Lambda$ we can make the identificarions $X_{2}^{\mu}(x)=\mu^{2} T(x)$ and $Y_{2}^{\mu}(x)=$ $\mu^{2} Y(x)$ familiar from the Argonne-Urbana two nucleon force expressions [33]. In the present case, however, $\Lambda_{\alpha} \neq \Lambda_{\beta}$ for each propagator and we must employ the longer forms 


$$
\begin{aligned}
X_{2 \alpha \beta}^{\mu}(x) & =\mu^{2}\left[F(\mu x)-\frac{\Lambda_{\beta}^{3}}{\mu^{3}}\left(\frac{\Lambda_{\alpha}^{2}-\mu^{2}}{\Lambda_{\alpha}^{2}-\Lambda_{\beta}^{2}}\right) F\left(\Lambda_{\beta} x\right)+\frac{\Lambda_{\alpha}^{3}}{\mu^{3}}\left(\frac{\Lambda_{\beta}^{2}-\mu^{2}}{\Lambda_{\alpha}^{2}-\Lambda_{\beta}^{2}}\right) F\left(\Lambda_{\alpha} x\right)\right], \\
Y_{2 \alpha \beta}^{\mu}(x) & =\mu^{2}\left[\frac{e^{-\mu x}}{\mu x}-\frac{\Lambda_{\beta}^{3}}{\mu^{3}}\left(\frac{\Lambda_{\alpha}^{2}-\mu^{2}}{\Lambda_{\alpha}^{2}-\Lambda_{\beta}^{2}}\right) \frac{e^{-\Lambda_{\beta} x}}{\Lambda_{\beta} x}+\frac{\Lambda_{\alpha}^{3}}{\mu^{3}}\left(\frac{\Lambda_{\beta}^{2}-\mu^{2}}{\Lambda_{\alpha}^{2}-\Lambda_{\beta}^{2}}\right) \frac{e^{-\Lambda_{\alpha} x}}{\Lambda_{\alpha} x}\right] .
\end{aligned}
$$

Before displaying the final potential in $r$-space let us introduce the "tilde" notation for the combination $1+\kappa_{\rho}$ which appears so often. For example, the $\rho$ propagator in (30) which has two form factors in the $\rho N N$ coupling and only one in the $\rho \Delta N$, takes on the compact form

$$
\tilde{Z}_{N G \rho}(x)=Z_{D G \rho}(x)+\kappa_{\rho} Z_{P G \rho}(x) .
$$

Since differentiation is a linear operator we can write, for example, .

$$
\tilde{X}_{2}^{\rho}(x) \equiv \tilde{X}_{2 N G}^{\rho}(x)=X_{2 D G}^{\rho}(x)+\kappa_{\rho} X_{2 P G}^{\rho}(x) .
$$

to shorten the following equations.

Using Eq. (31) the first term of Eq. (30) (from $\overrightarrow{\sigma_{1}} \cdot \vec{q} \overrightarrow{\sigma_{2}} \cdot \vec{q} \vec{k} \cdot \vec{k}$ ) becomes

$$
\begin{aligned}
\left.<\overrightarrow{r_{1}^{\prime}}, \overrightarrow{r_{2}^{\prime}}, \overrightarrow{r_{3}^{\prime}}\left|\mathcal{W}_{\rho \pi \Delta}^{+}(3 A)\right| \overrightarrow{r_{1}}, \overrightarrow{r_{2}}, \overrightarrow{r_{3}}\right\rangle_{1}= & \delta^{3}\left(\overrightarrow{x_{1}^{\prime}}-\overrightarrow{x_{1}}\right) \delta^{3}\left(\overrightarrow{x_{2}^{\prime}}-\overrightarrow{x_{2}}\right) \delta^{3}\left(\overrightarrow{x_{3}^{\prime}}-\overrightarrow{x_{3}}\right) \\
& \frac{-\mu m_{\rho}}{(4 \pi)^{2} 48 M m^{4}} g g_{\rho} \frac{5 M-m}{M-m} G_{M \rho}^{*}\left(m g^{*}\right) \\
& \overrightarrow{\tau_{1}} \cdot \overrightarrow{\tau_{2}}\left[\frac{\overrightarrow{\sigma_{1}} \cdot \overrightarrow{\sigma_{2}}}{3} \tilde{Y}_{2}^{\rho}\left(x_{13}\right) Y_{2}^{\mu}\left(x_{23}\right)\right. \\
& \left.\left.+\frac{S_{12}\left(\hat{x}_{23}\right)}{3} \tilde{Y}_{2}^{\rho}, x_{13}\right) X_{2}^{\mu}\left(x_{23}\right)\right]
\end{aligned}
$$

The second term of Eq. (30) has the same structure $\left(\overrightarrow{\sigma_{1}} \cdot \vec{k} \overrightarrow{\sigma_{2}} \cdot \vec{q} \vec{k} \cdot \vec{q}\right)$ as the "b" term of the $2 \pi$ exchange potential and is

$$
\begin{aligned}
\left.<\overrightarrow{r_{1}^{\prime}}, \overrightarrow{r_{2}^{\prime}}, \overrightarrow{r_{3}^{\prime}}\left|\mathcal{W}_{\rho \pi \Delta}^{+}(3 A)\right| \overrightarrow{r_{1}}, \overrightarrow{r_{2}}, \overrightarrow{r_{3}}\right\rangle_{2}= & \delta^{3}\left(\overrightarrow{x_{1}^{\prime}}-\overrightarrow{x_{1}}\right) \delta^{3}\left(\overrightarrow{x_{2}^{\prime}}-\overrightarrow{x_{2}}\right) \delta^{3}\left(\overrightarrow{x_{3}^{\prime}}-\overrightarrow{x_{3}}\right) \\
& \frac{+\mu m_{\rho}}{(4 \pi)^{2} 48 M m^{4}} g g_{\rho} \frac{5 M-m}{M-m} G_{M \rho}^{*}\left(m g^{*}\right) \\
& \overrightarrow{r_{1}} \cdot \overrightarrow{r_{2}} \frac{1}{9}\left[\overrightarrow{\sigma_{1}} \cdot \overrightarrow{\sigma_{2}} \tilde{Y}_{2}^{\rho}\left(x_{13}\right) Y_{2}^{\mu}\left(x_{23}\right)\right. \\
& +S_{12}\left(\hat{x}_{13}\right) \tilde{X}_{2}^{\rho}\left(x_{13}\right) Y_{2}^{\mu}\left(x_{23}\right)+S_{12}\left(\hat{x}_{23}\right) \tilde{Y}_{2}^{\rho}\left(x_{13}\right) X_{2}^{\mu}\left(x_{23}\right) \\
& +\left(9 \sigma_{1} \cdot \hat{x}_{13} \sigma_{2} \cdot \hat{x}_{23} \hat{x}_{13} \cdot \hat{x}_{23}-S_{12}\left(\hat{x}_{13}\right)-S_{12}\left(\hat{x}_{23}\right)\right. \\
& \left.\left.-\overrightarrow{\sigma_{1}} \cdot \overrightarrow{\sigma_{2}}\right) \tilde{X}_{2}^{\rho}\left(x_{13}\right) X_{2}^{\mu}\left(x_{23}\right)\right]
\end{aligned}
$$


The sum of these two terms agrees with the corresponding equation (2.23) of [3]. Identification of Eq. (38) with the coordinate space "b" term as given in ref. [33] can be made by remembering that $S_{12}\left(\hat{x}_{13}\right)=3 \overrightarrow{\sigma_{1}} \cdot \hat{x}_{13} \overrightarrow{\sigma_{2}} \cdot \hat{x}_{13}-\overrightarrow{\sigma_{1}} \cdot \overrightarrow{\sigma_{2}}$ and noting that $\left[Y_{2}(x)-X_{2}(x)\right] / 3=Z^{\prime}(x) / x$. The overall coordinate space constant (including the powers of meson masses in the definitions of $X_{2}^{\mu}(x)$ and $\left.Y_{2}^{\mu}(x)\right)$ for both terms (37) and (38) is $524.28 \mathrm{MeV}$. This number is huge because the $\rho N \Delta$ coupling constani $G_{M \rho}^{*}$ is (rather artifically) defined on the $\rho$ mass shell but is in reality obtained from the experimental $G_{M \gamma}^{*}\left(k^{2}=0\right)=14.7$. That is $G_{M \gamma}^{*}\left(k^{2}=0\right)=G_{M \rho}^{*} F_{\rho N \Delta}\left(k^{2}=0\right)$. Solving for $G_{M \rho}^{*}$, one finds $G_{M_{\rho}}^{*}=G_{M_{\gamma}}^{*}\left(k^{2}=0\right) \frac{\Lambda^{2}}{\Lambda^{2}-m_{\rho}^{2}}$ where $\Lambda=5.8 \mu$ and $m_{\rho} \approx 5.6 \mu$. The product $G_{M \rho}^{*} F_{\rho N \Delta}\left(k^{2}\right)$ is employed in the subthreshold region for space-like momentum transfer, where it is much smaller.

\subsubsection{Isospin odd amplitude}

Those la: gest terms $\mathrm{v} \quad$ ch are (t-channel) isospin odd multiply the spin functions of Eqs. (2.13f) and (2.23g) in Ref. [3]: , ${ }^{\prime} \mathcal{K}_{6}=i \overrightarrow{\sigma_{1}} \times \vec{k} \cdot \overrightarrow{\sigma_{3}} \overrightarrow{\sigma_{2}} \cdot \vec{q}$ and $m^{4} \mathcal{K}_{7}=i \overrightarrow{\sigma_{1}} \times \vec{k} \cdot \vec{q} \overrightarrow{\sigma_{3}} \cdot \vec{k} \overrightarrow{\sigma_{2}} \cdot \vec{q}$. The particular combination containing the delta-nucleon miss difference takes the form $m^{4} \mathcal{K}_{7}-\vec{q} \cdot \vec{k} m^{2} \mathcal{K}_{6}=$ $i \overrightarrow{\sigma_{1}} \times \vec{k} \cdot \vec{q} \overrightarrow{\sigma_{3}} \cdot \vec{k} \overrightarrow{\sigma_{2}} \cdot \vec{q}-\vec{q} \cdot \vec{k} i \overrightarrow{\sigma_{1}} \times \vec{k} \cdot \overrightarrow{\sigma_{3}} \overrightarrow{\sigma_{2}} \cdot \vec{q}$. This can be rewritten as $i \overrightarrow{\sigma_{1}} \times \vec{k} \cdot\left[\left(\overrightarrow{\sigma_{3}} \times \vec{q}\right) \times \vec{k}\right] \overrightarrow{\sigma_{2}} \cdot \vec{q}$ to show the spin structure connected with the several vertices most clearly, or finally as $-i\left(\overrightarrow{\sigma_{3}} \cdot \vec{q} \times \vec{k} \overrightarrow{\sigma_{1}}\right.$. $\left.\vec{k} \overrightarrow{\sigma_{2}} \cdot \vec{q}-\overrightarrow{\sigma_{1}} \times \overrightarrow{\sigma_{3}} \cdot \vec{q}(\vec{k})^{2} \overrightarrow{\sigma_{2}} \cdot \vec{q}\right)$. The third form is convenient for the coordinate space manipulations and has appeared before from non-relativistic transition potential derivations $[1,4]$. We will quote the momentum space potential with the last form of the spin functions

$$
\begin{aligned}
\left\langle\overrightarrow{p_{1}^{\prime}}, \overrightarrow{p_{2}^{\prime}}, \overrightarrow{p_{3}^{\prime}}\left|\mathcal{W}_{\rho \pi \Delta}^{-}(3 A)\right| \overrightarrow{p_{1}}, \overrightarrow{p_{2}}, \overrightarrow{p_{3}}\right\rangle= & -(2 \pi)^{3} \frac{\delta^{3}\left(\overrightarrow{p_{1}^{\prime}}+\overrightarrow{p_{2}^{\prime}}+\overrightarrow{p_{3}^{\prime}}-\overrightarrow{p_{1}}-\overrightarrow{p_{2}}-\overrightarrow{p_{3}}\right)}{\left(\vec{q}^{2}+\mu^{2}\right)\left(\overrightarrow{k^{2}}+m_{\rho}^{2}\right)} i \overrightarrow{\tau_{1}} \cdot \overrightarrow{\tau_{2}} \times \overrightarrow{\tau_{3}} \\
& i\left[\overrightarrow{\sigma_{3}} \cdot \vec{q} \times \vec{k} \overrightarrow{\sigma_{1}} \cdot \vec{k} \overrightarrow{\sigma_{2}} \cdot \vec{q}-\overrightarrow{\sigma_{1}} \times \overrightarrow{\sigma_{3}} \cdot \vec{q}(\vec{k})^{2} \overrightarrow{\sigma_{2}} \cdot \vec{q}\right] \\
& \frac{1}{96 m^{5}} g_{\rho}\left(F_{1 \rho}\left(k^{2}\right)+\kappa_{\rho} F_{2 \rho}\left(k^{2}\right)\right) G_{M \rho}^{*} F_{\rho N \Delta}\left(k^{2}\right) \\
& \frac{m}{M} \frac{M+m}{M-m} g F_{\pi N N}\left(q^{2}\right)\left(m g^{*} F_{\pi N \Delta}\left(q^{2}\right)\right)
\end{aligned}
$$

One can readily see that the first term has the same spin structure $\overrightarrow{\sigma_{3}} \cdot \vec{q} \times \vec{k} \overrightarrow{\sigma_{1}} \cdot \vec{k} \overrightarrow{\sigma_{2}} \cdot \vec{q}$ as the "d" term of the $2 \pi$ exchange potential. In coordinate space the isospin odd potential becomes

$$
\begin{aligned}
\left\langle\overrightarrow{r_{1}^{\prime}}, \overrightarrow{r_{2}^{\prime}}, \overrightarrow{r_{3}^{\prime}}\left|\mathcal{W}_{\rho \pi \Delta}^{-}(3 A)\right| \overrightarrow{r_{1}}, \overrightarrow{r_{2}}, \overrightarrow{r_{3}}\right\rangle= & \delta^{3}\left(\overrightarrow{x_{1}^{\prime}}-\overrightarrow{x_{1}}\right) \delta^{3}\left(\overrightarrow{x_{2}^{\prime}}-\overrightarrow{x_{2}}\right) \delta^{3}\left(\overrightarrow{x_{3}^{\prime}}-\overrightarrow{x_{3}}\right) \\
& \frac{\mu m_{\rho}}{(4 \pi)^{2} 96 M m^{4}} g g_{\rho} \frac{M+m}{M-m} G_{M \rho}^{*}\left(m g^{*}\right) \overrightarrow{\tau_{1}} \cdot \overrightarrow{\tau_{2}} \times \overrightarrow{\tau_{3}}
\end{aligned}
$$




$$
\begin{aligned}
& {\left[\overrightarrow{\sigma_{3}} \cdot \overrightarrow{\nabla_{2}} \times \overrightarrow{\nabla_{1}} \overrightarrow{\sigma_{1}} \cdot \overrightarrow{\nabla_{1}} \overrightarrow{\sigma_{2}} \cdot \overrightarrow{\nabla_{2}}-\overrightarrow{\sigma_{1}} \times \overrightarrow{\sigma_{3}} \cdot \overrightarrow{\nabla_{2}} \overrightarrow{\nabla_{1}} \cdot \overrightarrow{\nabla_{1}} \overrightarrow{\sigma_{2}} \cdot \overrightarrow{\nabla_{2}}\right]} \\
& \left(\left(Z_{D G \rho}\left(x_{13}\right)+\kappa_{\rho} Z_{P G \rho}\left(x_{13}\right)\right) Z_{N \Delta \mu}\left(x_{23}\right)\right.
\end{aligned}
$$

After the derivatives are carried out, the first term of Eq. (40), analogous to the "d" term of the $2 \pi$ exchange potential, takes the form:

$$
\begin{aligned}
<\overrightarrow{r_{1}^{\prime}}, \overrightarrow{r_{2}^{\prime}}, \overrightarrow{r_{3}^{\prime}}\left|\mathcal{W}_{\rho \pi \Delta}^{-}(3 A)\right| \overrightarrow{r_{1}}, \overrightarrow{r_{2}}, \overrightarrow{r_{3}}>_{1}= & \delta^{3}\left(\overrightarrow{x_{1}^{\prime}}-\overrightarrow{x_{1}}\right) \delta^{3}\left(\overrightarrow{x_{2}^{\prime}}-\overrightarrow{x_{2}}\right) \delta^{3}\left(\overrightarrow{x_{3}^{\prime}}-\overrightarrow{x_{3}}\right) \\
& \frac{\mu m_{\rho}}{(4 \pi)^{2} 96 M m^{4}} g g \rho \frac{M+m}{M-m} G_{M \rho}^{*}\left(m g^{*}\right) \\
& \overrightarrow{\tau_{1}} \cdot \overrightarrow{\tau_{2}} \times \overrightarrow{\tau_{3}}\left[\left(\overrightarrow{\sigma_{3}} \cdot \hat{x}_{23} \times \hat{x}_{13}\right)\left(\overrightarrow{\sigma_{1}} \cdot \hat{x}_{13}\right)\left(\overrightarrow{\sigma_{2}} \cdot \hat{x}_{23}\right)\right. \\
& \tilde{X}_{2}^{\rho}\left(x_{13}\right) X_{2}^{\mu}\left(x_{23}\right) \\
& +\left(\overrightarrow{\sigma_{1}} \cdot \hat{x}_{13}\right)\left(\overrightarrow{\sigma_{2}} \cdot \hat{x}_{13} \times \overrightarrow{\sigma_{3}}\right) \frac{1}{x_{23}} \tilde{X}_{2}^{\rho}\left(x_{13}\right) X_{1}^{\mu}\left(x_{23}\right) \\
& +\left(\overrightarrow{\sigma_{2}} \cdot \hat{x}_{23}\right)\left(\overrightarrow{\sigma_{3}} \cdot \hat{x}_{23} \times \overrightarrow{\sigma_{1}}\right) \frac{1}{x_{13}} \tilde{X}_{1}^{\rho}\left(x_{13}\right) X_{2}^{\mu}\left(x_{23}\right) \\
& \left.+\left(\overrightarrow{\sigma_{2}} \cdot \overrightarrow{\sigma_{1}} \times \overrightarrow{\sigma_{3}}\right) \frac{1}{x_{13}} \tilde{X}_{1}^{\rho}\left(x_{13}\right) \frac{1}{x_{23}} X_{1}^{\mu}\left(x_{23}\right)\right]
\end{aligned}
$$

The second term of Eq. (40) does not have a counterpart in the $2 \pi$ exchange potential but instead takes the simple form:

$$
\begin{aligned}
\left.<\overrightarrow{r_{1}^{\prime}}, \overrightarrow{r_{2}^{\prime}}, \overrightarrow{r_{3}^{\prime}}\left|\mathcal{W}_{\rho \pi \Delta}^{-}(3 A)\right| \overrightarrow{r_{1}}, \overrightarrow{r_{2}}, \overrightarrow{r_{3}}\right\rangle_{2}= & \delta^{3}\left(\overrightarrow{x_{1}^{\prime}}-\overrightarrow{x_{1}}\right) \delta^{3}\left(\overrightarrow{x_{2}^{\prime}}-\overrightarrow{x_{2}}\right) \delta^{3}\left(\overrightarrow{x_{3}^{\prime}}-\overrightarrow{x_{3}}\right) \\
& \frac{\mu m_{\rho}}{(4 \pi)^{2} 96 M m^{4}} g g_{\rho} \frac{M+m}{M-m} G_{M \rho}^{*}\left(m g^{*}\right) \\
& \overrightarrow{\tau_{1}} \cdot \overrightarrow{\tau_{2}} \times \overrightarrow{\tau_{3}}\left[+\left(\overrightarrow{\sigma_{2}} \cdot \hat{x}_{23}\right)\left(\overrightarrow{\sigma_{3}} \cdot \overrightarrow{\sigma_{1}} \times \hat{x}_{23}\right) \tilde{Y}_{2}^{\rho}\left(x_{13}\right) X_{2}^{\mu}\left(x_{23}\right)\right. \\
& \left.+\left(\overrightarrow{\sigma_{2}} \cdot \overrightarrow{\sigma_{3}} \times \overrightarrow{\sigma_{1}}\right) \frac{1}{x_{23}} \tilde{Y}_{2}^{\rho}\left(x_{13}\right) X_{1}^{\mu}\left(x_{23}\right)\right]
\end{aligned}
$$

The overall numerical constant in both (41) and (42) is $109 \mathrm{MeV}$, about a factor of five smaller than that of the isospin even term (for this reason this term was not included in the nuclear matter calculation of ref. [3]; in a trinucleon calculation however it may turn out to be meaningful). A model three-nucleon potential derived from the transition potential approach would have an exact ratio of $4: 1$ for the isospin even versus isospin odd contributions.

One local term proportional to $i\left[\overrightarrow{\sigma_{1}} \times \vec{k} \cdot \overrightarrow{\sigma_{3}} \overrightarrow{\sigma_{2}} \cdot \vec{q}\right] \vec{k} \cdot \vec{k}$ remains from the expansion of the covariant expression for the amplitude with an intermediate $\Delta$. The overall numerical constant is about a factor of ten smaller than that of (41) and (42) so we will not consider it further. It requires a third derivative of $Z$ or first derivative of $Y_{2}$ in coordinate space anyway, which eventually suppresses it further. 
The total rho-pi contribution is obtained from $\mathcal{W}^{\rho \pi}(3 A)$ by frst adding the contribution from $\mathcal{W}^{\rho \pi}(3 B)=\left(\mathcal{W}^{\rho \pi}(3 A): 1 \leftrightarrow 2\right)$ and then taking cyclic permutations of three "active" nucleons . 


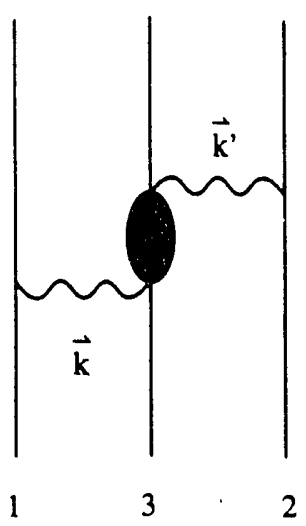

Figure 3: Diagram for the $\rho-\rho$ force

\section{The $\rho-\rho$ Potentials}

The definition of Eq. (3) is once more the starting point. The three-body $T_{N R}$ for the Feynman diagram of figure 3 involves the $\rho N \rightarrow \rho N$ amplitude, together with two $\rho$ meson propagators and $\rho \mathrm{NN}$ vertices.

The calculation of the latter amplitude is obtained by generalizing the Thirring theorem for Compton scattering to the case of $\rho$ mesons, through the current-field identity, under the assumption of vector dominance. The longitudinal character of the $\rho$ has however to be considered as well as the fact that it carries isospin. To do that one proceeds as did Bég [36] in his analysis of Compton scattering of isovector photons. The dominant term in the background (free of the nucleon pole term) Bég amplitude arises from the t channel $\rho$ pole ( $3 \rho$ coupling) and from a direct $\rho \rho \mathrm{NN}$ contact (seagull) term. Those contributions constitute the $\Delta T$ part of Eq. (20). The $\Delta$ term, contributing to the quadratic terms in the momenta, cannot be fixed by any divergence condition and is introduced, explicitely separated from the remaining amplitude, in a model dependent way. The following subsections will turn to the detailed form of all these components of the $\rho \mathrm{N}$ amplitude of different (physical) origin.

\subsection{Model-independent potentials from low-energy theorems}

The apparent dominant terms in the model-independent parts of this potential are odd in the tchannel isospin. The term displayed below results from the coupling of transverse (T) $\rho$-mesons to the low-energy amplitude constructed by Bég and has been labeled the "Bég" potential in the 
previous nuclear matter calculations. Similarly to the Kroll-Ruderman term, the "Bég" term is linear in each of the meson momentum, and it turns out to be important when comparing to the $\Delta$ isobar contributions. In momentum space it takes the form

$$
\begin{aligned}
\left\langle\overrightarrow{p_{1}^{\prime}}, \overrightarrow{p_{2}^{\prime}}, \overrightarrow{p_{3}^{\prime}}\left|W_{T T}^{-}\right| \overrightarrow{p_{1}}, \overrightarrow{p_{2}}, \overrightarrow{p_{3}}\right\rangle= & +(2 \pi)^{3} \frac{\delta^{3}\left(\overrightarrow{p_{1}^{\prime}}+\overrightarrow{p_{2}^{\prime}}+\overrightarrow{p_{3}^{\prime}}-\overrightarrow{p_{1}}-\overrightarrow{p_{2}}-\overrightarrow{p_{2}}\right)}{\left(\overrightarrow{k^{2}}+m_{\rho}^{2}\right)\left(\overrightarrow{k^{\prime}}+\overrightarrow{\tau_{\rho}^{2}}\right)} \times \overrightarrow{\tau_{3}} \\
& {\left[\left(\vec{\sigma}_{1} \times \vec{k}\right) \cdot\left(\overrightarrow{\sigma_{2}} \times \overrightarrow{k^{\prime}}\right) \times \vec{\sigma}_{3}\right] F_{1 \rho}^{2}(0)\left(1+\frac{F_{2 V}(0)}{F_{1 \rho}^{2}(0)}\right) } \\
& \frac{g_{\rho}^{4}}{64 m^{3}}\left(F_{1 \rho}\left(k^{2}\right)+\kappa_{\rho} F_{2 \rho}\left(k^{2}\right)\right)\left(F_{1 \rho}\left(k^{\prime 2}\right)+\kappa_{\rho} F_{2 \rho}\left(k^{\prime 2}\right)\right)
\end{aligned}
$$

where $F_{2 V}(0)=\kappa_{V}=3.7$ is the isovector anomalous moment of the nucleon. While the term independent of $F_{2 V}$ in $F_{1 \rho}^{2}(0)\left(1+\frac{F_{2 V}(0)}{F_{1 \rho}^{2}(0)}\right)$ arises from the backward-propagating nucleon pole term (or pair term), the term in $F_{2 V}(0)$ represents a direct $\rho \rho \mathrm{NN}$ contact (seagull) term in the underlying $\rho \mathrm{N} \rightarrow \rho \mathrm{N}$ amplitude. In principal the second term should be distinguished by a form factor of a different character $\left(F_{1 \rho}\left(\left(k-k^{\prime}\right)^{2}\right)\right.$ than the previous terms of the $\rho \pi$ potential. In practice, this component of the $\rho \rho$ potential has so far been calculated in the approximation of no form factors on the "active" nucleon and therefore includes only the $\rho \mathrm{NN}$ form factor at the external nucleons. This approximation is similar to the approximation made in the " $\mathrm{d}$ " term of the $2 \pi$ force. In that case, only the constant term of the current commutator expansion $F_{1 V}(t)+F_{2 V}(t) \approx$ $F_{1 V}(0)+F_{2 V}(0)+\mathcal{O}\left(q^{2}\right) \approx 1+3.70$ was retained (see Eq. (18)).

In coordinate space the "Bég" potential has a slightly different form from the previous ones displayed, because the propagator $\mathrm{Z}$ of Eq. has only a single monopole in the $\mathrm{H}$ function of Eq. (24) and (as in the "Kroll-Ruderman" term) only the first derivative is needed. With the definitions

$$
Z_{1 \mu}^{\prime} \equiv X_{11}^{\mu}(x)=-\mu\left[G(\mu x)-\frac{\Lambda^{2}}{\mu^{2}} G(\Lambda x)\right],
$$

where the added subscript 1 is meant to remind about the single formfactor, the coordinate space potential becomes

$$
\begin{aligned}
\left\langle\overrightarrow{r_{1}^{\prime}}, \overrightarrow{r_{2}^{\prime}}, \overrightarrow{r_{3}^{\prime}}\left|\mathcal{W}_{3 T T}^{(-)}\right| \overrightarrow{r_{1}}, \overrightarrow{r_{2}}, \overrightarrow{r_{3}}\right\rangle= & \delta^{3}\left(\overrightarrow{x_{1}^{\prime}}-\overrightarrow{x_{1}}\right) \delta^{3}\left(\overrightarrow{x_{2}^{\prime}}-\overrightarrow{x_{2}}\right) \delta^{3}\left(\overrightarrow{x_{3}^{\prime}}-\overrightarrow{x_{3}}\right) \\
& \frac{m_{\rho}^{2}}{(4 \pi)^{2} 64 m^{3}} g_{\rho}^{4} F_{1 \rho}^{2}(0)\left(1+\frac{F_{2 V}(0)}{F_{1 \rho}^{2}(0)}\right) \\
& \overrightarrow{r_{1}} \cdot \overrightarrow{r_{2}} \times \overrightarrow{r_{3}}\left(\overrightarrow{\sigma_{1}} \times \nabla_{1}\right) \cdot\left(\overrightarrow{\sigma_{2}} \times \nabla_{2}\right) \times \overrightarrow{\sigma_{3}} \\
& \dot{Z}_{1 m \cdot\left(x_{13}\right)} \tilde{Z}_{1 m_{\rho}}\left(x_{23}\right)
\end{aligned}
$$




$$
\begin{aligned}
\left\langle\overrightarrow{r_{1}^{\prime}}, \overrightarrow{r_{2}^{\prime}}, \overrightarrow{r_{3}^{\prime}}\left|\mathcal{W}_{3 T T}^{(-)}\right| \overrightarrow{r_{1}}, \overrightarrow{r_{2}}, \overrightarrow{r_{3}}\right\rangle= & \frac{m_{\rho}^{2}}{(4 \pi)^{2} 64 m^{3}} g_{\rho}^{4} F_{1 \rho}^{2}(0)\left(1+\frac{F_{2 V}(0)}{F_{1 \rho}^{2}(0)}\right) \\
& \delta^{3}\left(\overrightarrow{x_{1}^{\prime}}-\overrightarrow{x_{1}}\right) \delta^{3}\left(\overrightarrow{x_{2}^{\prime}}-\overrightarrow{x_{2}}\right) \delta^{3}\left(\overrightarrow{x_{3}^{\prime}}-\overrightarrow{x_{3}}\right) \\
& \overrightarrow{\tau_{1}} \cdot \overrightarrow{\tau_{2}} \times \overrightarrow{r_{3}}\left(\overrightarrow{\sigma_{1}} \times \hat{x}_{13}\right) \cdot\left(\overrightarrow{\sigma_{2}} \times \hat{x}_{23}\right) \times \overrightarrow{\sigma_{3}} \\
& \tilde{X}_{11}^{\rho}\left(x_{13}\right) \tilde{X}_{11}^{\rho}\left(x_{23}\right)
\end{aligned}
$$

\section{2 $\rho \rho$ Potential with $\Delta$ intermediate state}

There are two local potentials which correspond to the coupling of transverse $\rho$-mesons to the $\Delta$ pole. We introduce the notation

$$
\lambda_{M} \approx-\frac{3}{2 m(M+m)} G_{M \rho}^{*}
$$

for the low-momentum transfer $\rho \Delta \mathrm{N}$ vertex. The two local terms correspond to t-channel isospin even and odd amplitudes. We take them up in turn.

\subsubsection{Isospin even amplitude}

The form of the term generated by this amplitude agrees with the form arising from a transition potential construction and reads in momentum space

$$
\begin{aligned}
\left.<\overrightarrow{p_{1}^{\prime}}, \overrightarrow{p_{2}^{\prime}}, \overrightarrow{p_{3}^{\prime}}\left|W_{\rho \rho \Delta}^{+}\right| \overrightarrow{p_{1}}, \overrightarrow{p_{2}}, \overrightarrow{p_{3}}\right\rangle= & -(2 \pi)^{3} \frac{\delta^{3}\left(\overrightarrow{p_{1}^{\prime}}+\overrightarrow{p_{2}^{\prime}}+\overrightarrow{p_{3}^{\prime}}-\overrightarrow{p_{1}}-\overrightarrow{p_{2}}-\overrightarrow{p_{3}}\right)}{\left(\overrightarrow{k^{2}}+m_{\rho}^{2}\right)\left(\overrightarrow{k^{\prime}}{ }^{2}+m_{\rho}^{2}\right)} \\
& \overrightarrow{\tau_{1}} \cdot \overrightarrow{\tau_{2}}\left(\left(\overrightarrow{\sigma_{1}} \times \vec{k}\right) \times \vec{k}\right) \cdot\left(\left(\overrightarrow{\sigma_{2}} \times \overrightarrow{k^{\prime}}\right) \times \overrightarrow{k^{\prime}}\right) \\
& \frac{g_{\rho}^{2} \lambda_{M}^{2}}{18(M-m)}\left(F_{1 \rho}\left(k^{2}\right)+\kappa_{\rho} F_{2 \rho}\left(k^{2}\right)\right) F_{\rho N \Delta}\left(k^{2}\right) \\
& \left(F_{1 \rho}\left(k^{2}\right)+\kappa_{\rho} F_{2 \rho}\left(k^{2}\right)\right) F_{\rho N \Delta}\left(k^{\prime 2}\right)
\end{aligned}
$$

which, after Fourier transform becomes

$$
\begin{aligned}
\left\langle\overrightarrow{r_{1}^{\prime}}, \overrightarrow{r_{2}^{\prime}}, \overrightarrow{r_{3}^{\prime}}\left|\mathcal{W}_{\rho \rho \Delta}^{(+)}\right| \overrightarrow{r_{1}}, \overrightarrow{r_{2}} \cdot \overrightarrow{r_{3}}\right\rangle= & \delta^{3}\left(\overrightarrow{x_{1}^{\prime}}-\overrightarrow{x_{1}}\right) \delta^{3}\left(\overrightarrow{x_{2}^{\prime}}-\overrightarrow{x_{2}}\right) \delta^{3}\left(\overrightarrow{x_{3}^{\prime}}-\overrightarrow{x_{3}}\right) \\
& -\frac{m_{\rho}^{2}}{(4 \pi)^{2}} \frac{g_{\rho}^{2} \lambda_{M}^{2}}{18(M-m)} \overrightarrow{r_{1}} \cdot \overrightarrow{\tau_{2}}
\end{aligned}
$$




$$
\begin{aligned}
& {\left[\left(\left(\overrightarrow{\sigma_{1}} \times \vec{\nabla}_{1}\right) \times \vec{\nabla}_{1}\right) \cdot\left(\left(\overrightarrow{\sigma_{2}} \times \vec{\nabla}_{2}\right) \times \ddot{\nabla}_{2}\right)\right]} \\
& \left(Z_{D G \rho}\left(x_{13}\right)+\kappa_{\rho} Z_{P G \rho}\left(x_{13}\right)\right)\left(Z_{D G \rho}\left(x_{23}\right)+\kappa_{\rho} Z_{P G \rho}\left(x_{23}\right)\right)
\end{aligned}
$$

Carrying out the derivatives one obtains the final form of the matrix element in r-space

$$
\begin{aligned}
\left\langle\overrightarrow{r_{1}^{\prime}}, \overrightarrow{r_{2}^{\prime}}, \overrightarrow{r_{3}^{\prime}}\left|\mathcal{W}_{\rho \rho \Delta}^{(+)}\right| \overrightarrow{r_{1}}, \overrightarrow{r_{2}}, \overrightarrow{r_{3}}\right\rangle= & \delta^{3}\left(\overrightarrow{x_{1}^{\prime}}-\overrightarrow{x_{1}}\right) \delta^{3}\left(\overrightarrow{x_{2}^{\prime}}-\overrightarrow{x_{2}}\right) \delta^{3}\left(\overrightarrow{x_{3}^{\prime}}-\overrightarrow{x_{3}}\right) \\
& -\frac{m_{\rho}^{2}}{(4 \pi)^{2}} \frac{g_{\rho}^{2} \lambda_{M}^{2}}{18(M-m)} \overrightarrow{r_{1}} \cdot \overrightarrow{\tau_{2}} \frac{1}{9} \\
& \left(\left[4 \overrightarrow{\sigma_{1}} \cdot \overrightarrow{\sigma_{2}} \tilde{Y}_{2}^{\rho}\left(x_{13}\right) \tilde{Y}_{2}^{\rho}\left(x_{23}\right)-2 S_{12}\left(\hat{x}_{13}\right) \tilde{X}_{2}^{\rho}\left(x_{13}\right) \tilde{Y}_{2}^{\rho}\left(x_{23}\right)\right.\right. \\
& \left.-2 S_{12}\left(\hat{x}_{23}\right) \tilde{Y}_{2}^{\rho}\left(x_{13}\right) \tilde{X}_{2}^{\rho}\left(x_{23}\right)\right]+\left[9 \hat{x}_{13} \cdot \hat{x}_{23} \overrightarrow{\sigma_{1}} \cdot \hat{x}_{13} \overrightarrow{\sigma_{2}} \cdot \hat{x}_{23}\right. \\
& \left.\left.\left.-S_{12}\left(\hat{x}_{13}\right)-S_{12}\left(\hat{x}_{23}\right)-\overrightarrow{\sigma_{1}} \cdot \overrightarrow{\sigma_{2}}\right] \tilde{X}_{2}^{\rho}\left(x_{13}\right) \tilde{X}_{2}^{\rho}\left(x_{23}\right)\right]\right) .
\end{aligned}
$$

\subsubsection{Isospin odd amplitude}

There is a relative factor of $-1 / 4$ between isospin odd and isospin even in this case. This is precisely the relative factor obtained from a transition potential approach. The reason why the TM force ends up with the same factor lies in the fact that the $k \cdot C \cdot k^{\prime}$ contributions to the $\rho \mathrm{N} \rightarrow \rho \mathrm{N}$ amplitude were modeled by $\Delta$-poles in the s- and $\mathrm{u}$-channels.

$$
\begin{aligned}
\left.<\overrightarrow{p_{1}^{\prime}}, \overrightarrow{p_{2}^{\prime}}, \overrightarrow{p_{3}^{\prime}}\left|W_{\rho \rho \Delta}^{-}\right| \overrightarrow{p_{1}}, \overrightarrow{p_{2}}, \overrightarrow{p_{3}}\right\rangle= & +(2 \pi)^{3} \frac{\delta^{3}\left(\overrightarrow{p_{1}^{\prime}}+\overrightarrow{p_{2}^{\prime}}+\overrightarrow{p_{3}^{\prime}}-\overrightarrow{p_{1}}-\overrightarrow{p_{2}}-\overrightarrow{p_{3}}\right)}{\left(\vec{k}^{2}+m_{\rho}^{2}\right)\left(\overrightarrow{k^{\prime}}+m_{\rho}^{2}\right)} \\
& \frac{1}{4} \overrightarrow{\tau_{1}} \cdot \overrightarrow{\tau_{2}} \times \overrightarrow{\tau_{3}} \overrightarrow{\sigma_{3}} \cdot\left[\left(\left(\overrightarrow{\sigma_{1}} \times \vec{k}\right) \times \vec{k}\right) \times\left(\left(\overrightarrow{\sigma_{2}} \times \overrightarrow{k^{\prime}}\right) \times \overrightarrow{k^{\prime}}\right)\right] \\
& \frac{g_{\rho}^{2} \lambda_{M}^{2}}{18(M-m)}\left(F_{1 \rho}\left(k^{2}\right)+\kappa_{\rho} F_{2 \rho}\left(k^{2}\right)\right) F_{\rho N \Delta}\left(k^{2}\right) \\
& \left(F_{1 \rho}\left(k^{\prime 2}\right)+\kappa_{\rho} F_{2 \rho}\left(k^{\prime 2}\right)\right) F_{\rho N \Delta}\left(k^{2}\right)
\end{aligned}
$$

In coordinate space the equation becomes:

$$
\begin{aligned}
\left\langle\overrightarrow{r_{1}^{\prime}}, \overrightarrow{r_{2}^{\prime}}, \overrightarrow{r_{3}^{\prime}}\left|\mathcal{W}_{\rho \rho \Delta}^{(-)}\right| \overrightarrow{r_{1}}, \overrightarrow{r_{2}}, \overrightarrow{r_{3}}\right\rangle= & \delta^{3}\left(\overrightarrow{x_{1}^{\prime}}-\overrightarrow{x_{1}}\right) \delta^{3}\left(\overrightarrow{x_{2}^{\prime}}-\overrightarrow{x_{2}}\right) \delta^{3}\left(\overrightarrow{x_{3}^{\prime}}-\overrightarrow{x_{3}}\right) \\
& \frac{+m_{\rho}^{2}}{(4 \pi)^{2}} \frac{g_{\rho}^{2} \lambda_{M}^{2}}{18(M-m)} \frac{1}{4} \overrightarrow{\tau_{1}} \cdot \overrightarrow{\tau_{2}} \times \overrightarrow{\tau_{3}} \\
& \overrightarrow{\sigma_{3}} \cdot\left[\left(\left(\overrightarrow{\sigma_{1}} \times \overrightarrow{\nabla_{1}}\right) \times \overrightarrow{\nabla_{1}}\right) \times\left(\left(\overrightarrow{\sigma_{2}} \times \overrightarrow{\nabla_{2}}\right) \times \overrightarrow{\nabla_{2}}\right)\right] \\
& \left(Z_{D G \rho}\left(x_{13}\right)+\kappa_{\rho} Z_{P G_{\rho}}\left(x_{13}\right)\right)\left(Z_{D G_{\rho}}\left(x_{23}\right)+\kappa_{\rho} Z_{P G_{\rho}}\left(x_{23}\right)\right)(52)
\end{aligned}
$$


We note that the expansion of the vector products in the previous equation leads to four terms. one of which is identical to the " $\mathrm{d}$ " term of the $\pi-\pi$ force.

After carrying out the derivatives we find finally:

$$
\begin{aligned}
\left\langle\overrightarrow{r_{1}^{\prime}}, \overrightarrow{r_{2}^{\prime}}, \overrightarrow{r_{3}^{\prime}}\left|\mathcal{W}_{\rho \rho \Delta}^{(-)}\right| \overrightarrow{r_{1}}, \overrightarrow{r_{2}}, \overrightarrow{r_{3}}\right\rangle= & \delta^{3}\left(\overrightarrow{x_{1}^{\prime}}-\overrightarrow{x_{1}}\right) \delta^{3}\left(\overrightarrow{x_{2}^{\prime}}-\overrightarrow{x_{2}}\right) \delta^{3}\left(\overrightarrow{x_{3}^{\prime}}-\overrightarrow{x_{3}}\right) \\
& \frac{+m_{\rho}^{2}}{(4 \pi)^{2}} \frac{g_{\rho}^{2} \lambda_{M}^{2}}{18(M-m)} \frac{1}{4} \overrightarrow{r_{1}} \cdot \overrightarrow{r_{2}} \times \overrightarrow{r_{3}} \\
& {\left[\left(\overrightarrow{\sigma_{2}} \cdot \overrightarrow{\sigma_{3}} \times \overrightarrow{\sigma_{1}}\right) \tilde{Y}_{2}^{\rho}\left(x_{13}\right) \tilde{Y}_{2}^{\rho}\left(x_{23}\right)\right.} \\
& -\left(\overrightarrow{\sigma_{3}} \cdot \overrightarrow{\sigma_{1}} \times \hat{x}_{23}\right)\left(\overrightarrow{\sigma_{2}} \cdot \hat{x}_{23} \tilde{Y}_{2}^{\rho}\left(x_{13}\right) \tilde{X}_{2}^{\rho}\left(x_{23}\right)\right. \\
& -\left(\overrightarrow{\sigma_{1}} \cdot \overrightarrow{\sigma_{2}} \times \overrightarrow{\sigma_{3}}\right) \tilde{Y}_{2}^{\rho}\left(x_{13}\right) \frac{\tilde{X}_{1}^{\rho}\left(x_{23}\right)}{x_{23}} \\
& -\left(\overrightarrow{\sigma_{3}} \cdot \hat{x}_{13} \times \overrightarrow{\sigma_{2}}\right)\left(\overrightarrow{\sigma_{1}} \cdot \hat{x}_{13}\right) \tilde{X}_{2}^{\rho}\left(x_{13}\right) \tilde{Y}_{2}^{\rho}\left(x_{23}\right) \\
& \left.-\left(\overrightarrow{\sigma_{3}} \cdot \overrightarrow{\sigma_{1}} \times \overrightarrow{\sigma_{2}}\right) \frac{\tilde{X}_{1}^{\rho}\left(x_{13}\right)}{x_{13}} \tilde{Y}_{2}^{\rho}\left(x_{23}\right)\right] \\
& -\left[\left(\overrightarrow{\sigma_{3}} \cdot \hat{x}_{23} \times \hat{x}_{13}\right)\left(\overrightarrow{\sigma_{1}} \cdot \hat{x}_{13}\right)\left(\overrightarrow{\sigma_{2}} \cdot \hat{x}_{23}\right) \tilde{X}_{2}^{\rho}\left(x_{13}\right) \tilde{X}_{2}^{\rho}\left(x_{23}\right)\right. \\
& -\left(\overrightarrow{\sigma_{1}} \cdot \hat{x}_{13}\right)\left(\overrightarrow{\sigma_{2}} \cdot \hat{x}_{13} \times \overrightarrow{\sigma_{3}}\right) \frac{1}{x_{23}} \tilde{X}_{2}^{\rho}\left(x_{13}\right) \tilde{X}_{1}^{\rho}\left(x_{23}\right) \\
& -\left(\overrightarrow{\sigma_{2}} \cdot \hat{x}_{23}\right)\left(\overrightarrow{\sigma_{1}} \cdot \overrightarrow{\sigma_{3}} \times \hat{x}_{23}\right) \frac{\overrightarrow{X_{1}^{\rho}}\left(x_{13}\right)}{x_{13}} \tilde{X}_{2}^{\rho}\left(x_{23}\right) \\
& \left.-\left(\overrightarrow{\sigma_{2}} \cdot \overrightarrow{\sigma_{1}} \times \overrightarrow{\sigma_{3}}\right) \frac{\tilde{X}_{1}^{\rho}\left(x_{13}\right)}{x_{13}} \frac{\tilde{X}_{1}^{\rho}\left(x_{23}\right)}{x_{23}}\right]
\end{aligned}
$$

where the four last terms correspond to the " $\mathrm{d}$ " term analog piece of the matrix element.

\section{Recommended choice of parameters}

The set of numerical values for the coupling constants and the monopole form-factor regulator masses are compiled in Table II. The monopole formfactors are defined in Eqs. (23) and (24).

This set differs from table A2 of ref. [3] only in the value for the $\pi \mathrm{N} \Delta$ coupling constant, which should be $g^{*}=1.82 \mu^{-1}$, instead of the larger value of that table. Only this value is consistent with the $\pi \mathrm{N} \Delta$ coupling constant implicit in the $2 \pi$ exchange three-body force (see Appendix A of Ref. [5]) and with the value obtained by the Karlsruhe group [11] from $\pi \mathrm{N}$ scattering analysis. They used a fixed-t dispersion relation for the invariant amplitudes in order to determine the parameters of an "effective pole" which simulates $\Delta$-exchange at energies near and below threshold. The coupling constant so determined corresponds to the dimensionless coupling $f^{*}\left(f^{* 2} / 4 \pi=0.26\right)$ used by Martzolff et al [1], and differs from the Hannover group choice $f^{* 2} / 4 \pi=0.35$ for their 


\begin{tabular}{|c|c|c|}
\hline & $\begin{array}{l}g \\
g^{*} \\
\Lambda_{\pi N N} \\
\Lambda_{\pi N \Delta}\end{array}$ & $\begin{array}{r}13.4 \\
1.8 \mu^{-1} \\
5.8 \mu \\
5.8 \mu\end{array}$ \\
\hline \multicolumn{3}{|l|}{ for the $\rho$} \\
\hline & $g_{\rho}$ & $\begin{array}{r}5.3 \\
191.1\end{array}$ \\
\hline & $\Lambda_{\rho N N}($ Dirac $)$ & $12 \mu$ \\
\hline & $\Lambda_{\rho N N}(\mathrm{Pauli})$ & $7.4 \mu$ \\
\hline & $\Lambda_{\rho N \Delta}$ & $5.8 \mu$ \\
\hline
\end{tabular}

Ta?le 2: Couplings labeled by a generic $g$ and cut-off masses labeled by $\Lambda$. See Ref. 3 for complete definitions.

transition potential [37]. The latter value is derived from the $\Delta$-width, using the interaction term of the Lagrangian and "should not be used in application of pole term formulas near or bejow threshold" [38]. This latter value is $40 \%$ higher and therefore emphasizes the effects of the forward propagating isobar in the transition potential picture compared to the covariant isobar contribution to $\pi \mathrm{N}$ scattering.

The decision for the prescribed values of the remaining parameters was justified in ref. [3]. Briefly, we recall here that,

- the coupling constants concerning the $\pi$ come from low energy $\pi-N$ scattering c : ta. The vilue of $g=13.4$ has been challenged in recent years by partial wave analyses of ...ucleonnucleon scattering [39]. Much discussion has ensued on this still "isettled point.

- the cut-off value for the $\pi N \Delta$ form factor is taken to be the same as $\pi \mathrm{NN}$, which is suggested by data from neutrino-nucleon scattering $\left(p+\nu \rightarrow \Delta^{++}+\mu^{-}\right)$.

- for the $\rho$ meson, the choices made on the parameters were determined through vector meson dominance models (which ties the needed hadronic $\rho \Delta N$ coupling to the $\gamma \Delta N$ vertex) that are believed to be valid for processes involving low four-momentum transfer. We follow the experimental analyses of the $\gamma \Delta \mathrm{N}$ vertex and use a monopole form factor with cutoff mass $\Lambda_{\rho N \Delta}=5.8 \mu$ to approximate this vertex. As for the $\rho \mathrm{NN}$ vertex, it has two couplings: a direct Dirac coupl and the Pauli coupling to the anomalous magnetic moment of the nucleon. In the Tucs, elbourne prescription, the tio of the Pauli to Jirac coupling is 
allowed to vary with the four-momentum of the $\rho$. This choice appears to accommodate reasonably both the vector dominance value of $\kappa_{V}\left(k^{2}=0\right)=3.7$ and the on-mass-shell value of $\kappa_{\rho}\left(k^{2}=m_{\rho}^{2}\right)=6.6$ [35]. The two cutoff r.asses $\Lambda_{\text {Dirac }}=12 \mu$ and $\Lambda_{\text {Pauli }}=7.4 \mu$ are then determined from nucleon electromagnetic form factor data. Somewhat later, a group led by Gari [40] made a detailed fit to the electromagnetic form factors of the nucleon with the ansatz suggested already in [3] and came to a similar conclusion as [3]. What was not noticed by the authors of [3] and presumably of [40] was that the $k^{2}$ dependence of the Dirac and Pauli rho form factors away from the rho mass shell were also shown and even parameterized for spacelike momentum transfer by Höhler anc' Pietarinen in [35]. Indeed, the ratio of Pauli to Dirac coupling from the latter analysis does not vary much with $k^{2}$ and is 6.0 at $k^{2}=0$ [41]. It turns out that this form factor result for the $\rho \mathrm{NN}$ vertex is quite compatible with the data for the electromagnetic isovector nucleon form factors [42].

We next discuss the most varied aspect of the TM force: the fact that each user feels free to substitute his or her own choice for the recommended cut-off value of the monopole $\pi N N$ form factor. Its recommended value $(\Lambda=5.8 \mu \approx 810 \mathrm{MeV})$ is inspired by the $5 \%$ Goldberger-Treiman discrepancy which is consistent with a $3 \%$ decrease in the $\pi N N$ form factor from $q^{2}=m_{\pi}^{2}$ to $q^{2}=0$ [34]. The present understanding of the variation of the other quantities in the Goldberger-Treiman relation from the chiral limit to the chirally broken "real world", imply an "error bar" of $\pm 200 \mathrm{MeV}$ for the $\Lambda$ determination. This makes our choice in good agreement with the OPEP tail in many contemporary NN potentials: $\Lambda \approx 980 \mathrm{MeV}$ for Argonne V14 [43], $\Lambda=800 \mathrm{MeV}$ in the present day evolution of the Bonn OBEP potentials [44], and $\Lambda=800 \mathrm{MeV}$ for both $\pi$ and $\rho$ in the Bochum potential [4]. The $\pi \mathrm{NN}$ form factor $\Lambda \approx 1300 \mathrm{MeV}$ of the full Bonn potential [45] has always been inconsistent with evidence other than partial wave analysis of the NN system [34]; this difficulty is expected to be overcome if additional diagrams with correlated $\rho-\pi$ exchange is included into the full model [46]. On the other hand, the cutoff mass $\Lambda_{0}=965 \mathrm{MeV}$ in the exponential form factor of Nijmegen potentials [47] corresponds to $\Lambda=\sqrt{2} \Lambda_{0} \approx 1365 \mathrm{MeV}$ for a monopole form factor [39], rather like that of the full Bonn potential.

We should note that the current controversy over the value of the on-shell $\pi N N$ coupling $g$ affects the cutoff mass determination from the Goldberger-Treiman discrepancy $\Delta_{G T}=1-$ $m g_{A}(0) /\left(f_{\pi} g\right) \approx 0.05$; a smaller coupling constant implies a smaller discrepancy and a larger cutoff mass. But other analyses which isolate one-pion exchange via a Regge analysis of the charge exchange data $d \sigma /\left.d t\right|_{n p}$ minus $d \sigma /\left.d t\right|_{\bar{p} p}$ also find a $3 \%$ decrease in the $\pi N N$ form factor from $q^{2}=m_{\pi}^{2}$ to $q^{2}=0$ [34], indicating a certain robustness in the recommended value of the cutoff mass.

The recent consensus on a low mass cutoff for pion exchange highlights the point already 
emphasized by the Hokkaido group [25] and, in the modern context, by the São Paulo group [48]. The contact terms (those proportional to a coordinate-space $\delta$-function and its derivatives) are spread out with increasing importance as $\Lambda$ becomes smaller and the size of the nucleon grows. The dominant attitude so far was to consider that these contact terms, bringing the nucleon structure signature, should not be included in potential models. In contrast, the low cutoff mass of the $\pi \mathrm{NN}$ and $\rho \mathrm{NN}$ form factors of the Bochum $\mathrm{NN}$ potential, means that all mesons more massive than the $\rho$ are subsumed into contact terms. This reformulation of the traditional OBEP can still yield a satisfactory description of the $\mathrm{NN}$ data, although a $\chi^{2}$ analysis is needed for a definite judgment. The nice feature of the Bochum NN potential is that the small two-pion exchange term is highly suppressed, cut down by four powers of a rapidly varying cutoff function and partially replaced bi the contact terms. Weinberg obtains similar contact terms in his NN potential from a presumer four-fermion interaction [9].

We finish this section by returning to the subject of the $\rho N N$ formfactor. We have developed the formulas in momentum and coordinate space for two separate Dirac and Pauli formfactors of the monopole type. Clearly it is easy to specialize these general formulas to a single formfactor and then the choice of parameters is between the vector dominance value of $\kappa_{V}\left(k^{2}=0\right)=3.7$ expected for a narrow resonance and the on-mass-shell value of $\kappa_{\rho}\left(k^{2}=m_{\rho}^{2}\right)=6.6$ obtained from a dispersive analysis of $\pi \mathrm{N}$ scattering. From our current understanding of the the $\rho \mathrm{NN}$ vertex, a better but more difficult to implement form factor might be those (Dirac and Pauli) of Eq. 4.3 in [35] which goes into a monopole for high $k^{2}$ but has a more complicated structure at small $k^{2}$ near 0 . We suggest that future users of three-body forces might concentrate on the $\rho N N$ vertex rather than on the $\pi N N$ vertex, where the forr: actor of the Tucson-Melbourne three-nucleon potentials is not only determined by particle and leon-nucleon data but, in addition, is rather consistent with most of the realistic two-nucleon poientials.

\section{Numerical Results}

It is well-known that calculations with many recent "realistic" two-nucleon potentials (Argonne V14, Nijmegen potential or the folded diagram version of the full Bonn potential) produce underbinding of the the trinucleon bound state [49]. However, the same interactions, when taken in conjunction with the TM two-pion exchange three-body force, provide too much binding. There are overlapping and retarded pion exchange graphs not discussed in this paper which yield non-local terms [8]. These have not been numerically evaluated $\because *$. but are expected to be less important than the (already small) nucleon-antinucleon pair term: ?e $2 \pi$-TM potential and henceforth expected not to alter 
the binding defect problem.

To test the idea that the three-nucleon potentials of the two-meson exchange structure extended to include $\rho$-exchange act against the overbinding effect of the $2 \pi$-TM potential, we estimated perturbative contributions to the binding energy of the triton from the extended TM force described in detail in the previous sections.

The calculation was done using a wavefunction in the coordinate-space obtained by solving the Faddeev equations with the Malfliet-Tjon I-III potential [50]. We present the results in Table III, where the individual contributions from the different physical processes considered in the force are singled out. The contributions from the $\pi-\pi$ force are slightly different from preliminary results presented before [49] because in this paper we use the expansion coefficients of Table I. [51] We stress that the wavefunction used is "semi-realistic" at most (no NN p or $d$ waves are included in the Malfliet-Tjon I-III potential). The past history of results obtained with the $\pi-\pi$ force showed us already that any calculation with less than 18 three-body channels has to be considered with caution. The numbers given here were produced merely in the spirit of having a quick and hopefully qualitative information on the importance of the $\rho$ exchange in the three-nucleon force. A much more sophisticated calculation, which is out of the scope of this paper whose aim is restricted to provide the force to be used, will be reported [21, 22] soon.

From table III we conclude first that the $\rho$ exchange does indeed modify the effect of the $\pi-\pi$ force and that the effect goes in the (expected) direction of less binding. Since it has been fashionable to take the derived strengths of a nuclear force seriously, but to consider the mesonnucleon form factor cutoffs as adjustable parameters, we show in the columns labeled "Martzolff" a set of calculations which keep the strength constants of the Tucson-Melbourne three-body force but use the much heavier cutoff masses from Ref. [1] (we had to convert however their monopole parameterization of the product of two vertices to our dipole convention to find $\Lambda_{\pi}=10.6 \mu$ and $\Lambda_{\rho}=13.4 \mu$ for all couplings to N's or $\Delta$ 's). We note then that although the contributions of the $\pi-\pi$ are, as is well known, strongly cutoff dependent, the inclusion of the $\pi-\rho$ force supresses, at least in this triton model, much of that cutoff dependence. Finally, the results obtained for the TM force show a satisfying pattern of decreasing effect with increased mass of the exchanged meson. The same pattern does not exist in the pointlike nucleons (large cutoff masses) calculation of the rightmost columns. In any event, the addition of three-body forces due to $\rho$ exchange, especially the $\rho-\pi$ exchange force, does indeed counter the too strong attraction of the $\pi-\pi$ and seems to stabilize the total effect under variations in form factors. We await future tests of these tentative conclusions with some eagerness. 


\begin{tabular}{|c|c|c|c|c|c|c|c|c|c|}
\hline \multicolumn{2}{|c|}{ Exchanged mesons } & \multicolumn{4}{|c|}{ Tucson-Melbourne form factors } & \multicolumn{4}{|c|}{ "Martzolff" form factors } \\
\hline & & $\overline{T_{z}}$ & $\Delta \bar{T}$ & $q^{\prime} \cdot \bar{M}_{\Delta} \cdot q$ & Total & $\overline{T_{z}}$ & $\Delta T$ & $q^{\prime} \cdot \bar{M}_{\Delta} \cdot q$ & Total \\
\hline \multicolumn{10}{|l|}{$\pi-\pi$} \\
\hline & $T^{+}$ & 0.055 & .0 .409 & 0.090 & -0.264 & 0.011 & -0.363 & -0.257 & -0.609 \\
\hline & $T^{-}$ & -0.032 & -0.052 & -0.077 & -0.161 & -0.043 & -0.070 & -0.105 & -0.218 \\
\hline & Total & 0.023 & -0.461 & 0.013 & -0.425 & -0.032 & -0.433 & -0.362 & -0.827 \\
\hline \multicolumn{10}{|l|}{$\rho-\pi$} \\
\hline & $T^{+}$ & & & -0.022 & -0.022 & & & 0.261 & 0.261 \\
\hline & $T^{-}$ & 0.215 & & 0.090 & 0.090 & 0.342 & & 0.340 & 0.340 \\
\hline & Total & 0.215 & & 0.068 & 0.283 & 0.342 & & 0.601 & 0.943 \\
\hline \multicolumn{10}{|l|}{$\rho-\rho$} \\
\hline & $T^{+}$ & & & -0.005 & -0.005 & & & -0.101 & -0.101 \\
\hline & $T^{-}$ & 0.001 & 0.002 & -0.007 & -0.004 & 0.005 & 0.019 & -0.247 & -0.223 \\
\hline & Total & 0.001 & 0.002 & -0.012 & -0.009 & 0.005 & 0.019 & -0.348 & -0.324 \\
\hline TOTA & & & & & -0.151 & & & & -0.208 \\
\hline
\end{tabular}

Table 3: The three-body force contributions (in $\mathrm{MeV}$ ) to the triton binding energy. The contributions are arranged according to Eq. (20). Distinction between the isospin even and odd contributions is also shown. The calculation was made perturbatively with a (three-channel) Malfliet-Tjon I-III model wave function.

\section{Acknowledgements}

M. T. P. wants to thank the Theory Group at CEBAF for the hospitality granted during her stay. This work was suppor'ed in part by the National Science Foundation and by Junta Nacional de Investigação Científic: JNICT). We are especially grateful for a NATO Collaborative Research Grant which enabled us to undertake and finish this project. 


\section{References}

[1] M. Martzolff, B. Loiseau, and P. Grangé, Phys. Lett. 92B, 46 (1980).

[2] M. R. Robilotta and M. P. Isidro Filho, Nucl. Phys. A414, 394 (1984).

[3] R. G. Ellis, S. A. Coon, and B. H. J. McKellar, Nucl. Phys. A438, 631 (1985).

[4] S. Deister, M. F. Gari, W. Krümpelmann, and M. Mahlke, Few-Body Systems 10, 1 (1991).

[5] S. A. Coon, M. D. Scadron, P. C. McNamee, B. R. Barrett, D. W. E. Blatt and B. H. J. McKellar, Nucl. Phys. A317, 242 (1979).

[6] G. E. Brown, A. M. Green, and W. Gerace, Nucl. Phys. A115, 435 (1968).

[7] H. T. Coelho, D. K. Das, and M. R. Robilotta, Phys. Rev. 28C, 1812 (1983); a corrected version appears in M. R. Robilotta and H. T. Coelho, Nucl. Phys. A460, 645 (1986).

[8] S. A. Coon and J. L. Friar, Phys. Rev. C34, 1060 (1986).

[9] S. Weinberg, Phys. Lett. B251, 288(1990); Nucl.Phys. B363, 3(1991); Phys. Lett. B295, 114 (1992).

[10] D. Murphy and S. A. Coon, work in progress

[11] G. Höhler in Landolt-Börnstein, Vol. 9 b2 Pion-Nucleon Scattering, ed. H. Schopper (Springer, Berlin,1983).

[12] M. G. Olsson and E. T. Osypowski, Nucl. Phys. B101, 136 (1975).

[13] M. D. Scadron and L. R. Thebaud, Phys. Rev. D9, 1544 (1974); M. D. Scadron, Few Body Dynamics, editors A. N. Mitra, I. Slaus, V. S. Bhasin, V. K. Gupta, North Holland(1976), p. 325.

[14] S. A. Coon and M. D. Scadron, Jour. Phys. G: Nucl. Part. Phys. 18, 1923 (1992).

[15] J. T. MacMullen and M. D. Scadron, Phys. Rev. D20 1069 (1979); 1081 (1979).

[16] S. A. Coon and M. D. Scadron, Acta Physica Polonica, B24, 589 (1993).

[17] C. R. Chen, G. L. Payne, J. L. Friar, and B. F. Gibson, Phys. Rev. C33, 1740 (1986).

[18] T. Sasakawa and S. Ishikawa, Few-Body Systems 1, 3 (1986).

[19] T. Sasakawa, S. Ishikawa, Y. Wu, and T-Y. Saito, Phys. Rev. Lett. 68, 3503 (1992).

[20] A. Stadler and P. U. Sauer, Phys. Rev. C46, 26 (1992)

[21] A. Stadler, J. Adam, H. Henning, and P. U. Sauer, in preparation

[22] A. Stadler, Bull. Am. Phys. Soc. 38, 1013 (1993).

[23] W. Glöckle, H. Witała, and Th. Cornelius, Nucl. Phys. A508, 115c (1990). 
[24] H. Kamada and W. Glöckle, "On the inclusion of 3N-Forces into the 4N-Yakubovsky equations", Bochum preprint.

[25] M. Sato, Y. Akaishi, and H. Tanaka, Prog. Theo. Phys. Supp. 56, 76 (1974).

[26] S. A. Coon, J. G. Zabolitzky, and D. W. E. Blatt, Z. Physik A281, 137 (1977).

[27] J. Carlson, Phys. Rev. C38, 1879 (1988); Nucl. Phys. A508, 141c (1990).

[28] R. B. Wiringa, Phys. Rev. C43, 1585 (1991).

[29] S. A. Coon and W. Glöckle, Phys. Rev. C23 1790 (1981)

[30] S. Weinberg, Phys. Rev. Lett. 17, 616 (1966).

[31] G. E. Hite, R. J. Jacob, and M. D. Scadron, Phys. Rev. D14, 1306 (1976).

[32] M. T. Peña, H. Henning, and P. U. Sauer, Phys.Rev. C42, 850 (1990).

[33] J. Carlson, V. R. Pandharipande, and R. B. Wiringa Nucl. Phys. A401, 59 (1983).

[34] S. A. Coon and M. D. Scadron, Phys. Rev. C23, 1150 (1981); ibid C42, 2256 (1990); $\pi-\mathrm{N}$ Newsletter, 3, 90 (1991).

[35] G. Höhler and E. Pietarinen, Nucl. Phys. B95, 210 (1975).

[36] M. A. B. Bég, Phys. Rev. 150, 1276 (1966).

[37] Ch. Hajduk, P. U. Sauer and W. Strueve, Nucl. Phys. A405, 581 (1983)

[38] see page 563 of [11].

[39] V. Stoks, R. Timmermans, and J. J. de Swart, Phys. Rev. C47, 512 (1993).

[40] M. Gari and W. Kruempelmann, Phys. Rev. D45, 1817 (1992).

[41] G. Höhler, private communication.

[42] G. Höhler, et al. Nucl. Phys. B114, 505 (1976).

[43] B. L. Friman, V. R. Pandharipande, and R. B. Wiringa, Phys. Rev. Lett. 51, 763 (1983).

[44] J. Haidenbauer, K. Holinde, and A. W. Thomas, Phys. Rev. C45, 952 (1992).

[45] R. Machleidt, K. Holinde, and Ch. Elster, Phys. Rep. 149, 1 (1987).

[46] K. Holinde, private communication

[47] M. M. Nagels, T. A. Rijken, and J. J. de Swart, Phys. Rev. D17, 768 (1978).

[48] M. R. Robilotta and M. P. Isidro Filho, Nucl. Phys. A451, 581 (1986)

[49] S. A. Coon and M. T. Peña, Few-Body Systems, Suppl. 6, 242 (1992).

[50] J. L. Friar, B. F. Gibson, and G. L. Payne, Z. Phys. A301, 309 (1981).

[51] In addition, a mistake in the sign of the (miniscule) isospin odd $\rho-\rho$ amplitude contribution from $T_{Z}+\Delta T$ ("Beg") has been corrected since Ref. [49]. 

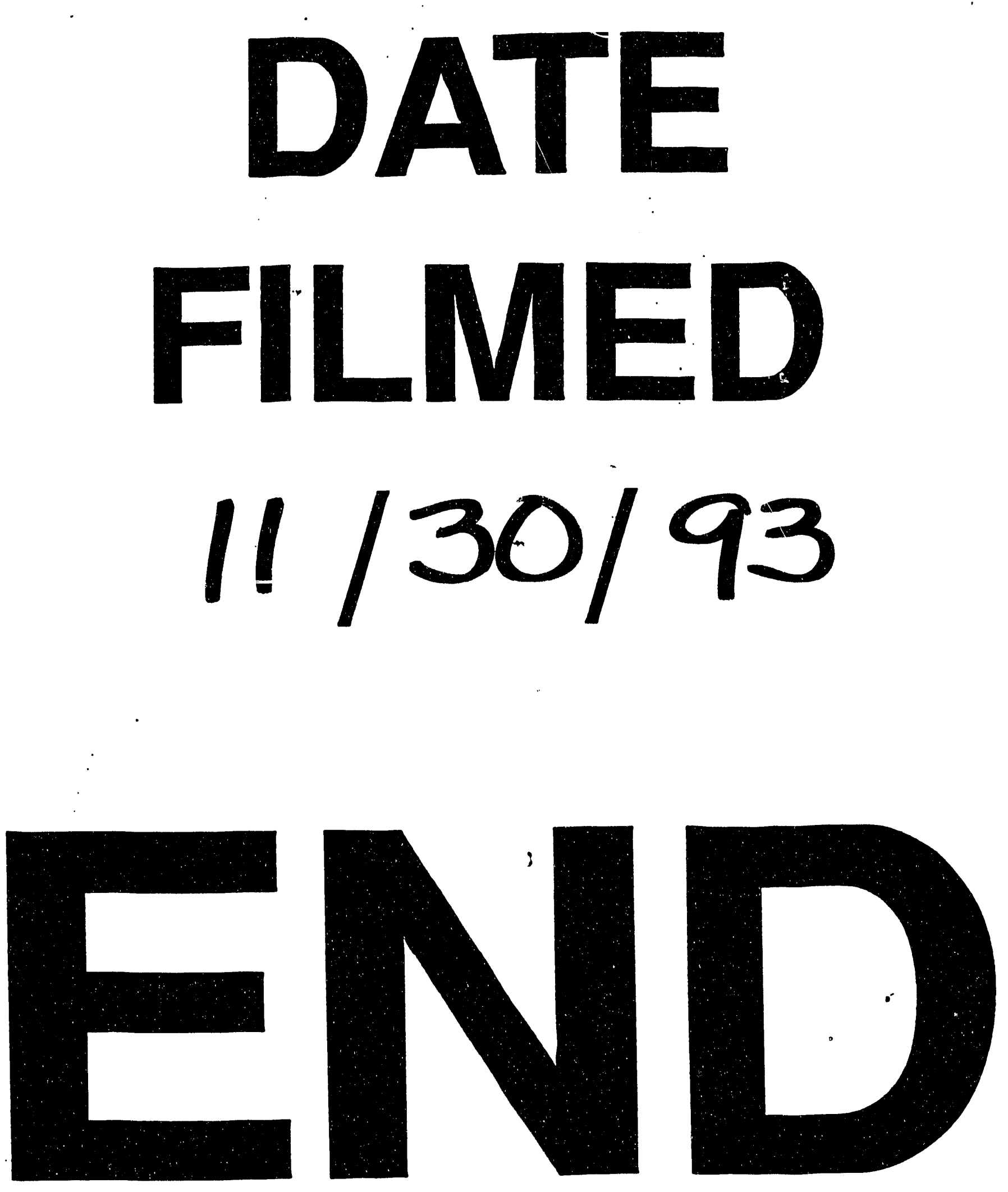
